\title{
The enclosure method for inverse obstacle scattering problems with dynamical data over a finite time interval II. Obstacles with a dissipative boundary or finite refractive index and back-scattering data
}

\author{
Masaru IKEHATA*
}

November 19, 2018

\begin{abstract}
In this paper a wave is generated by an initial data whose support is localized at the outside of unknown obstacles and observed in a limited time on a known closed surface or the same position as the support of the initial data. The observed data in the latter process are nothing but the back-scattering data. Two types of obstacles are considered. One is obstacles with a dissipative boundary condition which is a generalization of the sound-hard obstacles; another is obstacles with a finite refractive index, so-called, transparent obstacles. For each type of obstacles two formulae which yield explicitly the distance from the support of the initial data to unknown obstacles are given.

AMS: 35R30

KEY WORDS: enclosure method, inverse obstacle scattering problem, inverse backscattering, obstacle, wave equation, dissipative boundary condition, refractive index, transparent obstacle
\end{abstract}

\section{Introduction}

In [9] the author has introduced a simple method for the reconstruction issue of some class of inverse obstacle scattering problems. The observation data used therein are given by a wave field observed in limited time on a known closed surface surrounding unknown obstacles. The wave is generated by the initial data with compact support outside the surface. It is shown that the method yields the distance from a given point outside the surface to obstacles provided the obstacles are sound-hard or penetrable. It is a kind of an enclosure method since the obstacles can be considered as being contained in an open ball centered at $\infty$ determined by the distance.

The enclosure method was introduced in $[6,7]$ firstly for inverse boundary value problems governed by elliptic equations. In [8] the author has found its applications in several

${ }^{*}$ Department of Mathematics, Graduate School of Engineering, Gunma University, Kiryu 376-8515, JAPAN 
inverse initial boundary value problems governed by heat and wave equations in onespace dimensional case. Quite recently in $[11,12]$ the method has been extended to three-dimensional problems for the heat equation and in [10] the framework for inverse initial boundary value problem governed by the heat equation together with a result when the background medium is isotropic and inhomogeneous has been established.

This paper is a continuation of [9] and the purpose consists of two parts. First we extend the range of applications of this new method to more general or another kind of obstacles. We consider obstacles with a dissipative boundary condition which is a generalization of the sound-hard obstacles; obstacles with a finite refractive index, so-called, transparent obstacles. Second we consider so-called inverse back-scattering problems for those obstacles. Therein the observation data are measured in a limited time at the same position as the initial data. Needless to say this is a very important class of inverse problems for waves and the solution has many possibilities of applications, for example, nondestructive testing such as diffraction tomography, subsurface radar, microwave tomography, ocean acoustics tomography, etc. We show rigorously that the method works also for inverse back-scattering problems for obstacles mentioned above.

\subsection{Obstacle with a dissipative boundary condition}

First we consider an inverse obstacle scattering problem which is described by the classical wave equation outside an obstacle with a dissipative boundary condition.

Let $D$ be a nonempty bounded open subset of $\mathbf{R}^{3}$ with smooth boundary such that $\mathbf{R}^{3} \backslash \bar{D}$ is connected. Let $\gamma$ and $\beta$ be functions belonging to $L^{\infty}(\partial D)$ and satisfy $\gamma \geq 0$. Let $0<T<\infty$. Given $f \in L^{2}\left(\mathbf{R}^{3}\right)$ with compact support satisfying supp $f \cap \bar{D}=\emptyset$ let $u=u_{f}(x, t)$ denote the weak solution of the following initial boundary value problem for the classical wave equation:

$$
\begin{gathered}
\left.\partial_{t}^{2} u-\triangle u=0 \text { in }\left(\mathbf{R}^{3} \backslash \bar{D}\right) \times\right] 0, T[, \\
u(x, 0)=0 \text { in } \mathbf{R}^{3} \backslash \bar{D}, \\
\partial_{t} u(x, 0)=f(x) \text { in } \mathbf{R}^{3} \backslash \bar{D}, \\
\left.\frac{\partial u}{\partial \nu}-\gamma(x) \partial_{t} u-\beta(x) u=0 \text { on } \partial D \times\right] 0, T[.
\end{gathered}
$$

Here $\nu$ denotes the outward normal to $D$ on $\partial D$. Note that the boundary condition with $\beta \geq 0$ is called the dissipative boundary condition, however, in this paper we include also the case $\beta<0$ in this terminology. Note also that a formal computation yields

$$
E^{\prime}(t)=-2 \int_{\partial D} \gamma(x)\left|\partial_{t} u\right|^{2} d S \leq 0,
$$

where

$$
E(t)=\int_{\mathbf{R}^{3} \backslash \bar{D}}\left(\left|\partial_{t} u\right|^{2}+|\nabla u|^{2}\right) d x+\int_{\partial D} \beta(x)|u|^{2} d S, t \in[0, T] .
$$

Let $\Omega$ be a bounded domain of $\mathbf{R}^{3}$ with a smooth boundary such that $\bar{D} \subset \Omega$ and $\mathbf{R}^{3} \backslash \bar{\Omega}$ is connected. In this subsection we consider the following problem: 
Inverse Problem I. Assume that $D, \gamma$ and $\beta$ on $\partial D$ are unknown. Extract information about the location and shape of $D$ from the wave field $u_{f}(x, t)$ given at all $x \in \partial \Omega$ and $t \in] 0, T\left[\right.$ for a fixed $f \in L^{2}\left(\mathbf{R}^{3}\right)$ with compact support satisfying supp $f \cap \bar{\Omega}=\emptyset$.

Thus $\partial \Omega$ is a mathematical model of the closed surface on which the wave field is measured.

Let $B$ denote an open ball. In this paper as done in [7] we introduce two conditions on initial data $f$ :

(I1) $f(x)=0$ a.e. $x \in \mathbf{R}^{3} \backslash B$;

(I2) there exists a positive constant $C$ such that $f(x) \geq C$ a. e. $x \in B$ or $-f(x) \geq C$ a.e. $x \in B$.

Note that (I1) and (I2) imply that $\operatorname{supp} f=\bar{B}$.

Set

$$
w(x)=w_{f}(x, \tau)=\int_{0}^{T} e^{-\tau t} u_{f}(x, t) d t, x \in \mathbf{R}^{3} \backslash \bar{D}, \tau>0 .
$$

We denote by $\nu$ also the outward normal to $\Omega$ on $\partial \Omega$.

We assume that

- there exists a positive constant $C^{\prime}$ such that the one of the following two conditions is satisfied:

(A1) $\gamma(x) \leq 1-C^{\prime}$ a.e. $x \in \partial D$;

(A2) $\gamma(x) \geq 1+C^{\prime}$ a.e. $x \in \partial D$.

The first result of this subsection is the following formula which employs $w$ together with $\partial w / \partial \nu$ on $\partial \Omega$.

Theorem 1.1. Let $B$ satisfy $\bar{B} \cap \bar{\Omega}=\emptyset$ and $f \in L^{2}\left(\mathbf{R}^{3}\right)$ satisfy both (I1) and (I2). Let $v=v_{f}(\cdot, \tau) \in H^{1}\left(\mathbf{R}^{3}\right)$ denote the weak solution of the modified Helmholtz equation

$$
\left(\triangle-\tau^{2}\right) v+f=0 \text { in } \mathbf{R}^{3} .
$$

If the observation time $T$ satisfies

$$
T>2 \operatorname{dist}(D, B)-\operatorname{dist}(\Omega, B),
$$

then we have:

if (A1) is satisfied, then there exists a $\tau_{0}>0$ such that, for all $\tau \geq \tau_{0}$

$$
\int_{\partial \Omega}\left(\frac{\partial v}{\partial \nu} w-\frac{\partial w}{\partial \nu} v\right) d S>0
$$

if (A2) is satisfied, then then there exists a $\tau_{0}>0$ such that, for all $\tau \geq \tau_{0}$

$$
\int_{\partial \Omega}\left(\frac{\partial v}{\partial \nu} w-\frac{\partial w}{\partial \nu} v\right) d S<0
$$

In both cases the formula

$$
\lim _{\tau \longrightarrow \infty} \frac{1}{2 \tau} \log \left|\int_{\partial \Omega}\left(\frac{\partial v}{\partial \nu} w-\frac{\partial w}{\partial \nu} v\right) d S\right|=-\operatorname{dist}(D, B),
$$

is valid. 
Remark 1.1. $v$ in Theorem 1.1 is explicitly given by the formula

$$
v(x)=v_{f}(x, \tau)=\frac{1}{4 \pi} \int_{B} \frac{e^{-\tau|x-y|}}{|x-y|} f(y) d y
$$

and thus computable from $f$ in principle.

From the data $u_{f}(x, t)$ on $\left.\partial \Omega \times\right] 0, T[$ one can compute $w$ together with $\partial w / \partial \nu$ on $\partial \Omega$. The computation process makes use of the uniqueness of an initial boundary value problem for the wave equation in $\left.\left(\mathbf{R}^{3} \backslash \bar{\Omega}\right) \times\right] 0, T$. We do not repeat this description in detail and see [9] for the procedure. Thus formula (1.7) is: a solution to Inverse Problem I provided $\gamma$ satisfies (A1) or (A2); an extension of Theorem 1.1 in [9] which treated sound-hard obstacles, that is, the case when $\gamma=\beta=0$ on $\partial D$.

Note that dist $(D, B)+\sqrt{|\partial B| / 4 \pi}$ coincides with the distance from the center of $B$ to $D$. Thus by choosing a suitable initial data which is independent of $D$, from (1.7) one gets the distance of $D$ to a given point in $\mathbf{R}^{3} \backslash \bar{\Omega}$.

By Proposition 1.1. in [9] (1.4) ensures that $T>l(\partial B, \partial D, \partial \Omega)$, where

$$
l(\partial B, \partial D, \partial \Omega)=\inf \{|x-y|+|y-z| \mid x \in \partial B, y \in \partial D, z \in \partial \Omega\} .
$$

Thus (1.4) does not contradicts the finite propagation property of the signal governed by the wave equation. It should be emphasized that we never make use of the finite propagation property of the signal governed by the wave equation in any form and this is an interesting point of our method since we could find restriction (1.4).

It should be pointed out also that Theorem 1.1 gives a characterization whether $\gamma<1$ or $\gamma>1$ in terms of a solution of the wave equation over a finite time interval provided $\gamma$ is constant and $\gamma \neq 1$.

Our method covers also another very important case which employs the data observed on the support of the initial data not on $\partial \Omega$, that is, the back-scattering one. More precisely we consider the following problem.

Inverse Problem I'. Assume that $D, \gamma$ and $\beta$ on $\partial D$ are unknown. Let $B$ satisfy $\bar{B} \cap \bar{D}=\emptyset$. Extract information about the location and shape of $D$ from the wave field $u_{f}(x, t)$ given at all $x \in B$ and $\left.t \in\right] 0, T$ [ for a fixed $f \in L^{2}\left(\mathbf{R}^{3}\right)$ with support $\bar{B}$.

A new finding in the enclosure method what we want to emphasize is the following result.

Theorem 1.2. Let $B$ satisfy $\bar{B} \cap \bar{D}=\emptyset$ and $f \in L^{2}\left(\mathbf{R}^{3}\right)$ satisfy both (I1) and (I2). Let $v=v_{f}(\cdot, \tau) \in H^{1}\left(\mathbf{R}^{3}\right)$ be the weak solution of (1.3). If $T$ satisfies

$$
T>2 \operatorname{dist}(D, B),
$$

then we have:

if (A1) is satisfied, then there exists a $\tau_{0}>0$ such that, for all $\tau \geq \tau_{0}$

$$
\int_{B} f(w-v) d x>0
$$

if (A2) is satisfied, then there exists a $\tau_{0}>0$ such that, for all $\tau \geq \tau_{0}$

$$
\int_{B} f(w-v) d x<0
$$


In both cases the formula

$$
\lim _{\tau \longrightarrow \infty} \frac{1}{2 \tau} \log \left|\int_{B} f(w-v) d x\right|=-\operatorname{dist}(D, B),
$$

is valid.

In formula (1.12) we make use of $w(x, \tau)$ for $x \in B$ which can be computed directly from $u_{f}(x, t)$ for $\left.(x, t) \in B \times\right] 0, T[$ via (1.2). Since $\operatorname{supp} f=\bar{B}$, this is a back-scattering data over finite time interval ]0, $T$. Thus (1.12) gives a solution to Inverse Problem I'. Note also that $B$ can be arbitrary small.

Theorem 1.1 ensures that even the case when $T$ is not greater than 2 dist $(D, B)$, however satisfies (1.4) one can extract dist $(D, B)$ via formula (1.7). This is an expression of an advantage of making use of data $u_{f}$ on the whole of $\partial \Omega$. In back-scattering case, restriction (1.9) on $T$ is quite natural expected or optimal one because of the finite propagation property of the signal in the wave phenomena, however, note that in the proof we never make use of this property in any form.

In the framework of the Lax-Phiilips scattering theory [13], Majda [14] considered the case when $\beta=0$ and $D$ is strictly convex. He clarified the leading term of the scattering amplitude $s(\theta, \omega, \lambda)$ at high frequency which is the Fourier transform of the scattering kernel $S(s, \theta, \omega)$. However, our data are different from his ones and in this paper we never assume that $D$ is strictly convex.

\subsection{Obstacle with a finite refractive index}

Second we consider an inverse scattering problem for an obstacle with a finite refractive index.

Let $0<T<\infty$. Given $f \in L^{2}\left(\mathbf{R}^{3}\right)$ with compact support let $u=u_{f}(x, t)$ denote the weak solution of the following initial boundary value problem:

$$
\begin{aligned}
\alpha(x) \partial_{t}^{2} u-\triangle u & \left.=0 \text { in } \mathbf{R}^{3} \times\right] 0, T[, \\
u(x, 0) & =0 \text { in } \mathbf{R}^{3}, \\
\partial_{t} u(x, 0) & =f(x) \text { in } \mathbf{R}^{3},
\end{aligned}
$$

where $\alpha$ is a function belonging to $L^{\infty}\left(\mathbf{R}^{3}\right)$ and satisfies $\alpha(x) \geq C$ a.e. $x \in \mathbf{R}^{3}$ for a positive constant $C$.

We assume that

- there exists a nonempty bounded open set $D$ with a smooth boundary such that $\alpha(x)=1$ a.e. $x \in \mathbf{R}^{3} \backslash D$;

- there exists a positive constant $C^{\prime}$ such that the one of the following two conditions is satisfied:

(B1) $\alpha(x) \leq 1-C^{\prime}$ a.e. $x \in D$;

(B2) $\alpha(x) \geq 1+C^{\prime}$ a.e. $x \in D$.

Thus $\alpha$ has a jump across $\partial D$. $D$ is a mathematical model of an obstacle with a finite refractive index.

In this subsection we present a solution to the following problem. 
Inverse Problem II. Let $\Omega$ be a bounded domain of $\mathbf{R}^{3}$ with a smooth boundary such that $\bar{D} \subset \Omega$ and $\mathbf{R}^{3} \backslash \bar{\Omega}$ is connected. Assume that both $D$ and $\alpha$ in $D$ are unknown. Extract information about the location and shape of $D$ from the wave field $u_{f}(x, t)$ given at all $x \in \partial \Omega$ and $t \in] 0, T$ [ for a fixed $f \in L^{2}\left(\mathbf{R}^{3}\right)$ with compact support satisfying $\operatorname{supp} f \cap \bar{\Omega}=\emptyset$.

Set

$$
w(x)=w_{f}(x, \tau)=\int_{0}^{T} e^{-\tau t} u_{f}(x, t) d t, x \in \mathbf{R}^{3}, \tau>0 .
$$

Theorem 1.3. Let $B$ satisfy $\bar{B} \cap \bar{\Omega}=\emptyset$ and $f \in L^{2}\left(\mathbf{R}^{3}\right)$ satisfy both (I1) and (I2). Let $v=v_{f}(\cdot, \tau) \in H^{1}\left(\mathbf{R}^{3}\right)$ be the weak solution of (1.3).

If $T$ satisfy (1.4), then, we have:

if (B1) is satisfied, then there exists a $\tau_{0}>0$ such that, for all $\tau \geq \tau_{0}$

$$
\int_{\partial \Omega}\left(\frac{\partial v}{\partial \nu} w-\frac{\partial w}{\partial \nu} v\right) d S>0
$$

if (B2) is satisfied, then there exists a $\tau_{0}>0$ such that, for all $\tau \geq \tau_{0}$

$$
\int_{\partial \Omega}\left(\frac{\partial v}{\partial \nu} w-\frac{\partial w}{\partial \nu} v\right) d S<0
$$

In both cases we have

$$
\lim _{\tau \longrightarrow \infty} \frac{1}{2 \tau} \log \left|\int_{\partial \Omega}\left(\frac{\partial v}{\partial \nu} w-\frac{\partial w}{\partial \nu} v\right) d S\right|=-\operatorname{dist}(D, B) .
$$

The procedure for the computation of both $w$ and $\partial w / \partial \nu$ on $\partial \Omega$ from $u_{f}(x, t)$ given at all $x \in \partial \Omega$ and $t \in] 0, T$ [ is the same as Theorem 1.1. Thus this is a solution to Inverse Problem II.

The method works also for the following problem.

Inverse Problem II'. Assume that both $D$ and $\alpha$ in $D$ are unknown. Let $B$ satisfy $\bar{B} \cap \bar{D}=\emptyset$. Extract information about the location and shape of $D$ from the wave field $u_{f}(x, t)$ given at all $x \in B$ and $\left.t \in\right] 0, T$ [ for a fixed $f \in L^{2}\left(\mathbf{R}^{3}\right)$ with support $\bar{B}$.

The following result gives a solution to Inverse Problem II'.

Theorem 1.4. Let $B$ satisfy $\bar{B} \cap \bar{D}=\emptyset$ and $f \in L^{2}\left(\mathbf{R}^{3}\right)$ satisfy both (I1) and (I2). Let $v=v_{f}(\cdot, \tau) \in H^{1}\left(\mathbf{R}^{3}\right)$ be the weak solution of (1.3).

If $T$ satisfies (1.9), then we have:

if (B1) is satisfied, then there exists a $\tau_{0}>0$ such that, for all $\tau \geq \tau_{0}$

$$
\int_{B} f(w-v) d x>0
$$

if (B2) is satisfied, then there exists a $\tau_{0}>0$ such that, for all $\tau \geq \tau_{0}$

$$
\int_{B} f(w-v) d x<0
$$

In both cases we have

$$
\lim _{\tau \longrightarrow \infty} \frac{1}{2 \tau} \log \left|\int_{B} f(w-v) d x\right|=-\operatorname{dist}(D, B) .
$$


In the framework of the Lax-Phillips scattering theory there is a result by MajdaTaylor [15] for the case when $\alpha$ is smooth on $\bar{D}$ and $\alpha(x) \neq 1$. However, the data are given by the back-scattering kernel $S(s,-\omega, \omega), \omega \in S^{2}$ and the obtained information is the value of the support function of unknown obstacles and thus different from ours. For the study of the leading term of the scattering amplitude at high frequency for a strictly convex obstacle with a finite refractive index see Majda-Taylor [15] $(\alpha(x)>1$ on $\bar{D})$ and Petkov $[16,17](\alpha(x)<1$ on $\bar{D})$.

\subsection{Construction of the paper}

A brief outline of this paper is as follows. Theorems 1.1 and 1.2 are proved in Section 2. In Subsection 2 first we formulate what we mean by the weak solution of (1.1). It is based on the notion of the weak solution in [1] and an application of the existence theory therein. We see that $w$ given by (1.2) satisfies the modified Helmholtz equation with an unknown inhomogeneous term outside $D$ and boundary data on $\partial D$ in a weak sense. In Subsection 2.2 two expressions for the integral

$$
\int_{\partial \Omega}\left(\frac{\partial v}{\partial \nu} w-\frac{\partial w}{\partial \nu} v\right) d S
$$

are established. One is called the local expression which yields a bound of the absolute value of (1.21). Another is called the global expression and yields the lower bound of (1.21) in the case when (A1) is satisfied: upper bound of that in the case when (A2) is satisfied. Those bounds are presented in Subsections 2.4 and 2.5, respectively. Theorem 1.1 is a direct consequence of those bounds. The proof of Theorem 1.2 is described in Subsection 3.6. It is based on the following asymptotic formula which connects two data in Theorems 1.1 and 1.2 for an arbitrary fixed $T<\infty$ :

$$
\int_{\mathbf{R}^{3} \backslash \bar{D}} f(w-v) d x=\int_{\partial \Omega}\left(\frac{\partial v}{\partial \nu} w-\frac{\partial w}{\partial \nu} v\right) d S+O\left(\tau^{-1} e^{-\tau T}\right) .
$$

Using this together with the obtained bounds in Subsections 2.4 and 2.5, one gets immediately the conclusion of Theorem 1.2.

Section 3 is devoted to the proof of Theorems 1.3 and 1.4. The order of the presentation is parallel to that of Section 2. Starting with the formulation of the weak solution of (1.13), we present two expressions of (1.21) for $w$ given by (1.14). Both expressions are of global type since they involve integrals over the whole space. Using those expressions, we give a bound of the absolute value of(1.21) in Subsections 4.2; the upper/lower bound of (1.21) for case (B1)/(B2). Theorem 1.3 is a direct consequence of those bounds and an asymptotic formula which connects two data in Theorems 1.3 and 1.4 similar to (1.22) enables us to obtain all the conclusions of Theorem 1.4.

Section 4 consists of some remarks and open problems. In Subsection 4.1 we show that the case $\gamma=1$ is exceptional in one-space dimensional case. Moreover, the complete asymptotic expansion of (1.21) in one space-dimensional case is presented. In Subsection 4.2 corresponding to results in one-space dimensional case we propose some open problems and point out related results in some references together with future direction of our method. 


\section{Proof of Theorems 1.1 and 1.2}

\subsection{The weak solution and the governing equation for $w_{f}$}

The contents of this subsection almost parallel to the corresponding parts of Section 2 in [9] which is the case when $\gamma=\beta=0$ on $\partial D$.

We write $u^{\prime}$ instead of $\partial_{t} u$. It has been observed in [9] that for the enclosure method the notion of the weak solution described on pp 552-66 in [1] is appropriate since we consider only the solution on a finite time interval.

Set $V=H^{1}\left(\mathbf{R}^{3} \backslash \bar{D}\right)$ and $H=L^{2}\left(\mathbf{R}^{3} \backslash \bar{D}\right)$. Applying Theorem 1 on $\mathrm{p} 558$ in [1] to (1.1), we know that: given $u^{0} \in V$ and $u^{1} \in H$ there exists a unique $u$ satisfying

$$
u \in L^{2}(0, T ; V), u^{\prime} \in L^{2}(0, T ; V), u^{\prime \prime} \in L^{2}\left(0, T ; V^{\prime}\right)
$$

such that, for all $\phi \in V$

$$
\left.<u^{\prime \prime}(t), \phi>+a(u(t), \phi)+b\left(u^{\prime}(t), \phi\right)=0, \text { a.e. } t \in\right] 0, T[,
$$

and $u(0)=u^{0}$ and $u^{\prime}(0)=u^{1}$, where

$$
a(u, v)=\int_{\mathbf{R}^{3} \backslash \bar{D}} \nabla u \cdot \nabla v d x+\int_{\partial D} \beta u v d S, u, v \in V
$$

and

$$
b(u, v)=\int_{\partial D} \gamma u v d S, u, v \in V .
$$

Note that this is the case when $f=0$ in their notation and from their proof one knows that the restriction (5.8) on p 553 for $b$ is redundant in this case, that means that $b_{0}$ therein can be identically zero and $b=b_{1}$. Note that at this step we do not make use of condition $\gamma \geq 0$ on $\partial D$.

In this section we say that this $u=u_{f}$ for $u^{0}=0$ and $u^{1}=f$ is the weak solution of (1.1). Then $w=w_{f}$ given by (1.2) belongs to $V$; it follows from integration by parts and (2.1) multiplied by $e^{-\tau t}$ that $w$ satisfies for all $\phi \in V$

$$
\begin{gathered}
\int_{\mathbf{R}^{3} \backslash \bar{D}} \nabla w \cdot \nabla \phi d x+\int_{\partial D} c(x, \tau) w \phi d S+\int_{\mathbf{R}^{3} \backslash \bar{D}}\left(\tau^{2} w-f\right) \phi d x \\
=-e^{-\tau T} \int_{\mathbf{R}^{3} \backslash \bar{D}} F \phi d x-e^{-\tau T} \int_{\partial D} G \phi d S,
\end{gathered}
$$

where

$$
\begin{gathered}
c(x, \tau)=\gamma(x) \tau+\beta(x), \\
F(x, \tau)=u^{\prime}(x, T)+\tau u(x, T), \\
G(x)=\gamma(x) u(x, T) .
\end{gathered}
$$

This means that, in a weak sense, $w$ satisfies

$$
\begin{gathered}
\left(\triangle-\tau^{2}\right) w+f=e^{-\tau T} F(x, \tau) \text { in } \mathbf{R}^{3} \backslash \bar{D}, \\
\frac{\partial w}{\partial \nu}=c(x, \tau) w+e^{-\tau T} G(x) \text { on } \partial D .
\end{gathered}
$$


In particular, from (2.2) for $\phi \in C_{0}^{\infty}\left(\mathbf{R}^{3} \backslash \bar{D}\right)$, we have $\left(\triangle-\tau^{2}\right) w+f(x)=e^{-\tau T} F(x, \tau)$ in $\mathbf{R}^{3} \backslash \bar{D}$ in the sense of distribution and hence $\triangle w \in L^{2}\left(\mathbf{R}^{3} \backslash \bar{D}\right)$. This yields $w \in$ $H_{\text {loc }}^{2}\left(\mathbf{R}^{3} \backslash \bar{D}\right)$ and $\left(\triangle-\tau^{2}\right) w+f(x)=e^{-\tau T} F(x, \tau)$ a.e. $x \in \mathbf{R}^{3} \backslash \bar{D}$. Thus we can define $\partial w /\left.\partial \nu\right|_{\partial \Omega}$ as $\left.\nabla w\right|_{\partial \Omega} \cdot \nu \in H^{1 / 2}(\partial \Omega)$, where $\left.\nabla w\right|_{\partial \Omega}$ is the trace of $\nabla w$ onto $\partial \Omega([4])$.

\subsection{Local and global expressions of (1.21)}

In the following proposition we do not assume that $\operatorname{supp} f \cap \bar{D}=\emptyset$ nor $\operatorname{supp} f \cap \bar{\Omega}=\emptyset$.

Proposition 2.1. We have two expressions:

$$
\begin{gathered}
\int_{\partial \Omega}\left(\frac{\partial v}{\partial \nu} w-\frac{\partial w}{\partial \nu} v\right) d S \\
=\int_{\partial D}\left(\frac{\partial v}{\partial \nu}-c(x, \tau) v\right) w d S-\int_{\Omega \backslash \bar{D}}(w-v) f d x \\
-e^{-\tau T} \int_{\partial D} G v d S-e^{-\tau T} \int_{\Omega \backslash \bar{D}} F v d x \\
\int_{\partial \Omega}\left(\frac{\partial v}{\partial \nu} w-\frac{\partial w}{\partial \nu} v\right) d S \\
=\int_{D}\left(|\nabla v|^{2}+\tau^{2}|v|^{2}\right) d x-\int_{\partial D} c(x, \tau)|v|^{2} d S-e^{-\tau T} \int_{\partial D} G v d S \\
+\int_{\mathbf{R}^{3} \backslash \bar{D}}\left\{|\nabla(w-v)|^{2}+\tau^{2}|w-v|^{2}\right\} d x+\int_{\partial D} c(x, \tau)|w-v|^{2} d S \\
+e^{-\tau T} \int_{\mathbf{R}^{3} \backslash \bar{D}} F(w-v) d x-e^{-\tau T} \int_{\Omega \backslash \bar{D}} F v d x+e^{-\tau T} \int_{\partial D} G(w-v) d S \\
-\int_{\Omega \backslash \bar{D}}(w-v) f d x-\int_{D} f v d x .
\end{gathered}
$$

Proof. First we give a proof of (2.3). Let $\varphi \in H^{1}(\Omega \backslash \bar{D})$ satisfy $\varphi=0$ on $\partial \Omega$ in the sense of the trace. Since the zero extension to $\mathbf{R}^{3} \backslash \bar{D}$ of this $\varphi$ belongs to $V([4])$, we have from (2.2)

$$
\begin{gathered}
0=\int_{\Omega \backslash \bar{D}} \nabla w \cdot \nabla \varphi d x+\int_{\partial D} c(x, \tau) w \varphi d S \\
+\int_{\Omega \backslash \bar{D}}\left(\tau^{2} w-f+e^{-\tau T} F\right) \varphi d x+e^{-\tau T} \int_{\partial D} G \varphi d S .
\end{gathered}
$$

Choose $\chi \in C_{0}^{\infty}\left(\mathbf{R}^{3}\right)$ such that $\chi=1$ in a neighbourhood of $\partial \Omega$ and $\chi(x) \equiv 0$ in a neighbourhood of $\bar{D}$. Since $\left.(1-\chi) v\right|_{\Omega \backslash \bar{D}}$ vanishes in a neighbourhood of $\partial \Omega$, (2.5) for $\varphi=\left.(1-\chi) v\right|_{\Omega \backslash \bar{D}}$ is valid.

On the other hand, since $\chi v$ vanishes in a neighbourhood of $\bar{D}$ and $w \in H_{\text {loc }}^{2}\left(\mathbf{R}^{3} \backslash \bar{D}\right)$, 
integration by parts yields

$$
\begin{gathered}
\int_{\Omega \backslash \bar{D}} \nabla w \cdot \nabla(\chi v) d x=\int_{\partial \Omega} \frac{\partial w}{\partial \nu} \chi v d S-\int_{\Omega \backslash \bar{D}}(\Delta w) \chi v d x \\
=\int_{\partial \Omega} \frac{\partial w}{\partial \nu} v d S-\int_{\Omega \backslash \bar{D}}\left(\tau^{2} w-f+e^{-\tau T} F\right) \chi v d x
\end{gathered}
$$

that is

$$
\int_{\partial \Omega} \frac{\partial w}{\partial \nu} v d S=\int_{\Omega \backslash \bar{D}} \nabla w \cdot \nabla(\chi v) d x+\int_{\Omega \backslash \bar{D}}\left(\tau^{2} w-f+e^{-\tau T} F\right) \chi v d x .
$$

From this and $(2.5)$ for $\varphi=\left.(1-\chi) v\right|_{\Omega \backslash \bar{D}}$ which satisfies $\varphi=v$ on $\partial D$ we obtain

$$
\begin{gathered}
\int_{\partial \Omega} \frac{\partial w}{\partial \nu} v d S=\int_{\Omega \backslash \bar{D}} \nabla w \cdot \nabla v d x+\int_{\partial D} c(x, \tau) w v d S \\
+\int_{\Omega \backslash \bar{D}}\left(\tau^{2} w-f+e^{-\tau T} F\right) v d x+\int_{\partial D} e^{-\tau T} G v d S .
\end{gathered}
$$

By the trace theorem one can choose $\tilde{w} \in H^{1}(\Omega)$ such that $\tilde{w}=w$ in $\Omega \backslash \bar{D}$. Note that $\tilde{w}=w$ on $\partial \Omega$ and $\partial D$ in the sense of the trace. Since $v \in H^{2}(\Omega)$, we have

$$
\begin{gathered}
\int_{\partial \Omega} \frac{\partial v}{\partial \nu} w d S=\int_{\partial \Omega} \frac{\partial v}{\partial \nu} \tilde{w} d S \\
=\int_{\Omega} \Delta v \tilde{w} d x+\int_{\Omega} \nabla v \cdot \nabla \tilde{w} d x \\
=\int_{\Omega \backslash \bar{D}}\left(\tau^{2} v-f\right) w d x+\int_{\Omega \backslash \bar{D}} \nabla v \cdot \nabla w d x \\
+\int_{D}\left(\tau^{2} v-f\right) \tilde{w} d x+\int_{D} \nabla v \cdot \nabla \tilde{w} d x .
\end{gathered}
$$

On the other hand we have

$$
\begin{gathered}
\int_{D} \nabla v \cdot \nabla \tilde{w} d x=\int_{\partial D} \frac{\partial v}{\partial \nu} \tilde{w} d S-\int_{D}(\triangle v) \tilde{w} d x \\
=\int_{\partial D} \frac{\partial v}{\partial \nu} w d S-\int_{D}\left(\tau^{2} v-f\right) \tilde{w} d x
\end{gathered}
$$

that is,

$$
\int_{\partial D} \frac{\partial v}{\partial \nu} w d S=\int_{D}\left(\tau^{2} v-f\right) \tilde{w} d x+\int_{D} \nabla v \cdot \nabla \tilde{w} d x
$$

Therefore, we obtain

$$
\int_{\partial \Omega} \frac{\partial v}{\partial \nu} w d S=\int_{\Omega \backslash \bar{D}}\left(\tau^{2} v-f\right) w d x+\int_{\Omega \backslash \bar{D}} \nabla v \cdot \nabla w d x+\int_{\partial D} \frac{\partial v}{\partial \nu} w d S
$$

A combination of (2.6) and (2.7) gives (2.3). 
Next we give a proof of (2.4). Write

$$
\int_{\partial D} \frac{\partial v}{\partial \nu} w d S=\int_{\partial D} \frac{\partial v}{\partial \nu}(w-v) d S+\int_{\partial D} \frac{\partial v}{\partial \nu} v d S
$$

By the trace theorem given $\phi \in V$ one can choose $\tilde{\phi} \in H^{1}\left(\mathbf{R}^{3}\right)$ such that $\phi=\left.\tilde{\phi}\right|_{\mathbf{R}^{3} \backslash \bar{D}}$. Since $v \in H^{2}(D)$ and $\triangle v-\tau^{2} v+f=0$ a.e. $x \in D$ and $\phi=\tilde{\phi}$ on $\partial D$ in the sense of the trace, integration by parts yields

$$
\int_{\partial D} \frac{\partial v}{\partial \nu} \phi d S=\int_{D}\left(\nabla v \cdot \nabla \tilde{\phi} d x+\tau^{2} v \tilde{\phi}\right) d x-\int_{D} f \tilde{\phi} d x
$$

On the other hand $v$ satisfies

$$
-\int_{\mathbf{R}^{3}} \nabla v \cdot \nabla \varphi d x-\tau^{2} \int_{\mathbf{R}^{3}} v \varphi d x=-\int_{\mathbf{R}^{3}} f \varphi d x, \forall \varphi \in H^{1}\left(\mathbf{R}^{3}\right) .
$$

Substituting $\varphi=\tilde{\phi}$ into this identity and dividing $\mathbf{R}^{3}=D \cup\left(\mathbf{R}^{3} \backslash D\right)$, we obtain

$$
\int_{\mathbf{R}^{3} \backslash \bar{D}}\left(\nabla v \cdot \nabla \phi+\tau^{2} v \phi\right) d x=\int_{\mathbf{R}^{3} \backslash \bar{D}} f \phi d x-\int_{D}\left(\nabla v \cdot \nabla \tilde{\phi}+\tau^{2} v \tilde{\phi}\right) d x+\int_{D} f \tilde{\phi} d x .
$$

A combination of this and (2.9) gives, for all $\phi \in V$,

$$
\int_{\partial D} \frac{\partial v}{\partial \nu} \phi d S+\int_{\mathbf{R}^{3} \backslash \bar{D}}\left(\nabla v \cdot \nabla \phi+\tau^{2} v \phi\right) d x=\int_{\mathbf{R}^{3} \backslash \bar{D}} f \phi d x .
$$

Combining this with (2.2), we obtain

$$
\begin{gathered}
\int_{\partial D}\left(\frac{\partial v}{\partial \nu}-c(x, \tau) v\right) \phi d S=\int_{\partial D} c(x, \tau) \epsilon \phi d S+\int_{\mathbf{R}^{3} \backslash \bar{D}}\left\{\nabla \epsilon \cdot \nabla \phi+\tau^{2} \epsilon \phi\right\} d x \\
+e^{-\tau T} \int_{\mathbf{R}^{3} \backslash \bar{D}} F \phi d x+e^{-\tau T} \int_{\partial D} G \phi d S,
\end{gathered}
$$

where $\epsilon=w-v$. This means that $\epsilon$ satisfies, in a weak sense

$$
\begin{gathered}
\left(\triangle-\tau^{2}\right) \epsilon=e^{-\tau T} F \text { in } \mathbf{R}^{3} \backslash \bar{D} \\
\frac{\partial \epsilon}{\partial \nu}-c(x, \tau) \epsilon=-\frac{\partial v}{\partial \nu}+c(x, \tau) v+e^{-\tau T} G \text { on } \partial D .
\end{gathered}
$$

Substituting $\epsilon$ for $\phi$ in (2.11), we obtain

$$
\begin{aligned}
\int_{\partial D} \frac{\partial v}{\partial \nu} \epsilon d S= & \int_{\partial D} c(x, \tau)\left(|\epsilon|^{2}+v \epsilon\right) d S+\int_{\mathbf{R}^{3} \backslash \bar{D}}\left(|\nabla \epsilon|^{2}+\tau^{2}|\epsilon|^{2}\right) d x \\
& +e^{-\tau T} \int_{\mathbf{R}^{3} \backslash \bar{D}} F \epsilon d x+e^{-\tau T} \int_{\partial D} G \epsilon d S
\end{aligned}
$$

Now from this together with $(2,3),(2.8)$ and the identities $v \epsilon=-|v|^{2}+v w$ and

$$
\int_{\partial D} \frac{\partial v}{\partial \nu} v d S=\int_{D}\left(|\nabla v|^{2}+\tau^{2}|v|^{2}\right) d x-\int_{D} f v d x
$$


we obtain (2.4).

Now assume that supp $f \cap \bar{\Omega}=\emptyset$. From (2.3) and (2.4) we have the following two expressions which we call the local expression and global expression, respectively:

$$
\begin{gathered}
\int_{\partial \Omega}\left(\frac{\partial v}{\partial \nu} w-\frac{\partial w}{\partial \nu} v\right) d S \\
=\int_{\partial D}\left(\frac{\partial v}{\partial \nu}-c(x, \tau) v\right) w d S-e^{-\tau T} \int_{\partial D} G v d S-e^{-\tau T} \int_{\Omega \backslash \bar{D}} F v d x \\
\int_{\partial \Omega}\left(\frac{\partial v}{\partial \nu} w-\frac{\partial w}{\partial \nu} v\right) d S \\
=\int_{D}\left(|\nabla v|^{2}+\tau^{2}|v|^{2}\right) d x-\int_{\partial D} c(x, \tau)|v|^{2} d S-e^{-\tau T} \int_{\partial D} G v d S \\
+\int_{\mathbf{R}^{3} \backslash \bar{D}}\left\{|\nabla(w-v)|^{2}+\tau^{2}|w-v|^{2}\right\} d x+\int_{\partial D} c(x, \tau)|w-v|^{2} d S \\
+e^{-\tau T} \int_{\mathbf{R}^{3} \backslash \bar{D}} F(w-v) d x-e^{-\tau T} \int_{\Omega \backslash \bar{D}} F v d x+e^{-\tau T} \int_{\partial D} G(w-v) d S .
\end{gathered}
$$

For convenience we set

$$
I(\tau)=\int_{\partial \Omega}\left(\frac{\partial v}{\partial \nu} w-\frac{\partial w}{\partial \nu} v\right) d S
$$

We make use of (2.14) to give an estimation of $|I(\tau)|$ from above and (2.15) of $I(\tau)$ from below/above when $(\mathrm{A} 1) /(\mathrm{A} 2)$ is satisfied. This is the role of (2.14) and (2.15).

\subsection{An estimate of $|I(\tau)|$ from above}

In this subsection we derive the following estimate as $\tau \longrightarrow \infty$ :

$$
e^{2 \tau \operatorname{dist}(D, B)}|I(\tau)|=O\left(\tau^{3 / 2}\right) .
$$

Lemma 2.1. Let $\epsilon=w-v$. As $\tau \longrightarrow \infty$ we have

$$
\|\epsilon\|_{L^{2}\left(\mathbf{R}^{3} \backslash \bar{D}\right)}^{2}=O\left(e^{-2 \tau T}+e^{-2 \tau \operatorname{dist}(D, B)}\right)
$$

and

$$
\|\nabla \epsilon\|_{L^{2}\left(\mathbf{R}^{3} \backslash \bar{D}\right)}^{2}=O\left(\tau^{2}\left(e^{-2 \tau T}+e^{-2 \tau \operatorname{dist}(D, B)}\right)\right) .
$$

Proof. Rewrite (1.12) as

$$
\begin{gathered}
\int_{\mathbf{R}^{3} \backslash \bar{D}}\left(|\nabla \epsilon|^{2}+\tau^{2}|\epsilon|^{2}\right) d x=-\int_{\partial D} c(x, \tau)\left(|\epsilon|^{2}+v \epsilon\right) d S+\int_{\partial D} \frac{\partial v}{\partial \nu} \epsilon d S \\
-e^{-\tau T}\left(\int_{\mathbf{R}^{3} \backslash \bar{D}} F \epsilon d x+\int_{\partial D} G \epsilon d S\right) .
\end{gathered}
$$


We have

$$
\begin{gathered}
-\int_{\partial D} c(x, \tau)\left(|\epsilon|^{2}+v \epsilon\right) d S+\int_{\partial D} \frac{\partial v}{\partial \nu} \epsilon d S \\
=-\tau \int_{\partial D} \gamma\left(|\epsilon|^{2}+v \epsilon\right) d S+\int_{\partial D}\left(\frac{\partial v}{\partial \nu}-\beta|\epsilon|^{2}-\beta v \epsilon\right) d S
\end{gathered}
$$

Completing the square and $\gamma \geq 0$ on $\partial D$ give

$$
\begin{aligned}
-\int_{\partial D} \gamma\left(|\epsilon|^{2}+v \epsilon\right) d S & =-\int_{\partial D} \gamma\left|\epsilon+\frac{v}{2}\right|^{2} d S+\int_{\partial D} \frac{\gamma}{4}|v|^{2} d S \\
& \leq \int_{\partial D} \frac{\gamma}{4}|v|^{2} d S .
\end{aligned}
$$

Moreover we have

$$
\begin{gathered}
\int_{\partial D}\left(\frac{\partial v}{\partial \nu} \epsilon-\beta|\epsilon|^{2}-\beta v \epsilon\right) d S \leq C \int_{\partial D}\left(|\epsilon|^{2}+|v|^{2}+\left|\frac{\partial v}{\partial \nu}\right|^{2}\right) d S \\
e^{-\tau T}\left|\int_{\partial D} G \epsilon d S\right| \leq \frac{1}{2}\left(\int_{\partial D}|\epsilon|^{2} d S+e^{-2 \tau T} \int_{\partial D}|G|^{2} d S\right)
\end{gathered}
$$

and

$$
e^{-\tau T}\left|\int_{\mathbf{R}^{3} \backslash \bar{D}} F \epsilon d x\right| \leq \frac{1}{2}\left(\eta^{2} \int_{\mathbf{R}^{3} \backslash \bar{D}}|\epsilon|^{2} d x+e^{-2 \tau T} \eta^{-2} \int_{\mathbf{R}^{3} \backslash \bar{D}}|F|^{2} d x\right), \forall \eta>0 .
$$

From (2.19)-(2.24) we obtain

$$
\begin{gathered}
\int_{\mathbf{R}^{3} \backslash \bar{D}}|\nabla \epsilon|^{2} d x+\left(\tau^{2}-\frac{\eta^{2}}{2}\right) \int_{\mathbf{R}^{3} \backslash \bar{D}}|\epsilon|^{2} d x \\
\leq\left(C+\frac{1}{2}\right) \int_{\partial D}|\epsilon|^{2} d S+\int_{\partial D}\left(C+\frac{\tau \gamma}{4}\right)|v|^{2} d S+C \int_{\partial D}\left|\frac{\partial v}{\partial \nu}\right|^{2} d S \\
+\frac{e^{-2 \tau T}}{2}\left(\int_{\partial D}|G|^{2} d S+\frac{1}{2 \eta^{2}} \int_{\mathbf{R}^{3} \backslash \bar{D}}|F|^{2} d x\right) .
\end{gathered}
$$

Here we cite the well known inequality (e.g., [4]) that there exists a positive constant $K=K(\Omega \backslash \bar{D})$ such that, for all $z \in H^{1}(\Omega \backslash \bar{D})$

$$
\int_{\partial D}|z|^{2} d S \leq K\left(\eta^{2} \int_{\Omega \backslash \bar{D}}|\nabla z|^{2} d x+\eta^{-2} \int_{\Omega \backslash \bar{D}}|z|^{2} d x\right), \forall \eta>0 .
$$


Applying (2.26) to the first term in the right-hand side of (2.25), we have

$$
\begin{gathered}
\int_{\mathbf{R}^{3} \backslash \bar{\Omega}}|\nabla \epsilon|^{2} d x+\left(\tau^{2}-\frac{\eta^{2}}{2}\right) \int_{\mathbf{R}^{3} \backslash \bar{\Omega}}|\epsilon|^{2} d x \\
+\left\{1-K \eta^{2}\left(C+\frac{1}{2}\right)\right\} \int_{\Omega \backslash \bar{D}}|\nabla \epsilon|^{2} d x+\left\{\left(\tau^{2}-\frac{\eta^{2}}{2}\right)-K \eta^{-2}\left(C+\frac{1}{2}\right)\right\} \int_{\Omega \backslash \bar{D}}|\epsilon|^{2} d x \\
\leq \int_{\partial D}\left\{\left(C+\frac{\tau \gamma}{4}\right)|v|^{2}+C\left|\frac{\partial v}{\partial \nu}\right|^{2}\right\} d S+\frac{e^{-2 \tau T}}{2}\left(\int_{\partial D}|G|^{2} d S+\frac{1}{2 \eta^{2}} \int_{\mathbf{R}^{3} \backslash \bar{D}}|F|^{2} d x\right) .
\end{gathered}
$$

Now choosing a small $\eta$, from (1.8) and (2.27) we obtain (2.17) and (2.18) as $\tau \longrightarrow \infty$.

Now from $(2.26)$ for $\eta=1 / \sqrt{\tau},(2.17)$ and (2.18) we obtain

$$
\|\epsilon\|_{L^{2}(\partial D)}^{2}=O\left(\tau\left(e^{-2 \tau T}+e^{-2 \tau \operatorname{dist}(D, B)}\right)\right)
$$

This together with $\|v\|_{L^{2}(\partial D)}=O\left(e^{-\tau \operatorname{dist}(D, B)}\right)$ yields

$$
\|w\|_{L^{2}(\partial D)}^{2}=O\left(\tau\left(e^{-2 \tau T}+e^{-2 \tau \operatorname{dist}(D, B)}\right)\right)
$$

Since

it follows from (2.29) that

$$
\left\|\frac{\partial v}{\partial \nu}\right\|_{L^{2}(\partial D)}=O\left(\tau e^{-\tau \operatorname{dist}(D, B)}\right)
$$

$$
\left|\int_{\partial D} \frac{\partial v}{\partial \nu} w d S\right|+\left|\int_{\partial D} c(x, \tau) v w d S\right|=O\left(\tau^{3 / 2}\left(e^{-2 \tau \operatorname{dist}(D, B)}+e^{-\tau(T+\operatorname{dist}(D, B)}\right)\right) .
$$

Moreover we have

$$
e^{-\tau T}\left|\int_{\partial D} G v d S\right|=O\left(e^{-\tau(T+\operatorname{dist}(D, B))}\right)
$$

and since

we obtain

$$
\|v\|_{L^{2}(\Omega \backslash \bar{D})}=O\left(e^{-\tau \operatorname{dist}(\Omega, B)}\right)
$$

$$
e^{-\tau T}\left|\int_{\Omega \backslash \bar{D}} F v d x\right|=O\left(\tau e^{-\tau(T+\operatorname{dist}(\Omega, B))}\right) .
$$

Now from $(2.14),(2.30),(2.31)$ and (2.32) we obtain

$$
|I(\tau)|=O\left(\tau^{3 / 2}\left(e^{-2 \tau \operatorname{dist}(D, B)}+e^{-\tau(T+\operatorname{dist}(D, B))}\right)+\tau e^{-\tau(T+\operatorname{dist}(\Omega, B))}\right)
$$

and thus

$$
e^{2 \tau \operatorname{dist}(D, B)}|I(\tau)|=O\left(\tau^{3 / 2}\left(1+e^{-\tau(T-\operatorname{dist}(D, B))}\right)+\tau e^{-\tau(T-2 \operatorname{dist}(D, B)+\operatorname{dist}(\Omega, B))}\right) .
$$

Write

$$
T-\operatorname{dist}(D, B)=(T-2 \operatorname{dist}(D, B)+\operatorname{dist}(\Omega, B))+(\operatorname{dist}(D, B)-\operatorname{dist}(\Omega, B)) .
$$

This together with $\operatorname{dist}(D, B)>\operatorname{dist}(\Omega, B)$ and $(1.4)$ gives $T>\operatorname{dist}(D, B)$. Now from this together with (1.4) and (2.33) we obtain (2.16).

Remark 2.1. From this last part we know that, for the proof of (2.16) it suffice to assume only $T \geq 2 \operatorname{dist}(D, B)-\operatorname{dist}(\Omega, B)$ instead of stricter condition (1.4). 


\subsection{Case (A1). An estimate of $I(\tau)$ from below}

In this subsection we prove that: there exists a positive constant $\mu$ such that

$$
\liminf _{\tau \longrightarrow \infty} \tau^{\mu} e^{2 \tau \operatorname{dist}(D, B)} I(\tau)>0
$$

provided $\gamma$ satisfies (A1).

Having (2.16) and (2.34), we immediately obtain (1.5) and (1.7).

For the proof of (2.34) first we study the asymptotic behaviour of the following integral

$$
J(\tau) \equiv \int_{D}\left(|\nabla v|^{2}+\tau^{2}|v|^{2}\right) d x-\int_{\partial D} c(x, \tau)|v|^{2} d S_{x} .
$$

Since $\bar{B} \cap \bar{D}=\emptyset, f(x)=0$ a.e. $x \in D$ and thus (2.13) gives another expression:

$$
J(\tau)=\int_{\partial D}\left(\frac{\partial v}{\partial \nu}-c(x, \tau) v\right) v d S
$$

Lemma 2.2. There exists a positive number $\mu$ such that

$$
\lim _{\tau \longrightarrow \infty} \tau^{\mu} e^{2 \tau \operatorname{dist}(D, B)} J(\tau)>0 .
$$

Proof. Since

$$
\frac{\partial v}{\partial \nu}(x)=-\frac{\tau}{4 \pi} \int_{B} \frac{(x-y) \cdot \nu(x)}{|x-y|^{2}} e^{-\tau|x-y|} f(y) d y-\frac{1}{4 \pi} \int_{B} \frac{(x-y) \cdot \nu(x)}{|x-y|^{3}} e^{-\tau|x-y|} f(y) d y,
$$

we have

$$
J(\tau)=\frac{\tau}{(4 \pi)^{2}} \int_{\partial D} d S_{x} \int_{B \times B}\left(k(x, y)-\frac{1}{\tau} l(x, y)\right) \frac{e^{-\tau\left(|x-y|+\left|x-y^{\prime}\right|\right)}}{|x-y|\left|x-y^{\prime}\right|} f(y) f\left(y^{\prime}\right) d y d y^{\prime}
$$

where

$$
\begin{aligned}
& k(x, y)=\frac{(y-x) \cdot \nu(x)}{|x-y|}-\gamma(x), \\
& l(x, y)=\frac{(y-x) \cdot \nu(x)}{|x-y|^{2}}-\beta(x) .
\end{aligned}
$$

We divide the integrand of $J(\tau)$ into two parts. Set $d=\operatorname{dist}(\partial D, \bar{B})$ and $\mathcal{M}=$ $\{(x, y) \in \partial D \times \bar{B}|| x-y \mid=d\}$. It is easy to see that $d=\operatorname{dist}(D, B)$.

In what follows we denote by $B_{R}(z)$ the open ball centered at a point $z$ with radius $R$. Given $\delta>0$ define

$$
\mathcal{W}_{\delta}=\cup_{\left(x_{0}, y_{0}\right) \in \mathcal{M}}\left(\partial D \cap B_{\delta}\left(x_{0}\right)\right) \times\left(\bar{B} \cap B_{\delta}\left(y_{0}\right)\right) \times\left(\bar{B} \cap B_{\delta}\left(y_{0}\right)\right) .
$$

The set $\mathcal{W}_{\delta}$ is open in $\partial D \times \bar{B} \times \bar{B}$ and contains the set of all $(x, y, y)$ with $(x, y) \in \mathcal{M}$. Here we state the following two claims concerning the $\mathcal{W}_{\delta}$. Their proofs are almost same as those of Claims 1 and 2 in [12]. Just rewrite the proofs by replacing $\bar{D}$ and $\partial \Omega$ in the previous definitions of $\mathcal{M}, \mathcal{W}_{\delta}$ and $F\left(x, y, y^{\prime}\right)$ in [12] with $\partial D$ and $\bar{B} ;\left(x-y^{\prime}\right) /\left|x-y^{\prime}\right|$ 
in the previous definition of $F\left(x, y, y^{\prime}\right)$ in [12] with $\nu(x)$. By this reason we omit the description.

Claim 1. Given $\epsilon>0$ there exists a $\delta_{1}>0$ such that for all $\left(x, y, y^{\prime}\right) \in \mathcal{W}_{\delta_{1}}$ it holds that

$$
\frac{(y-x) \cdot \nu(x)}{|x-y|} \geq 1-\epsilon,|x-y| \leq d+\epsilon,\left|x-y^{\prime}\right| \leq d+\epsilon .
$$

Claim 2. Given $\delta_{1}>0$ there exists a $\delta_{2}>0$ such that if $\left(x, y, y^{\prime}\right) \in \partial D \times \bar{B} \times \bar{B} \backslash \mathcal{W}_{\delta_{1}}$, then $|x-y|+\left|x-y^{\prime}\right| \geq 2 d+\delta_{2}$.

Let $C^{\prime}$ be the constant in (A1). Give $\epsilon=C^{\prime} / 2$ in Claim 1 and choose $\delta_{1}$ in Claim 1 . Next choose $\delta_{2}$ in Claim 2 corresponding to $\delta_{1}$ already chosen.

By claim 1, we have, for all $\left(x, y, y^{\prime}\right) \in \mathcal{W}_{\delta_{1}} k(x, y) \geq 1-\epsilon-\left(1-C^{\prime}\right)=C^{\prime} / 2$, $|x-y| \leq d+\epsilon$ and $\left|x-y^{\prime}\right| \leq d+\epsilon$; by claim 2, we have $e^{-\tau\left(|x-y|+\left|x-y^{\prime}\right|\right)} \leq e^{-2 \tau d} e^{-2 \tau \delta_{2}}$ if $\left(x, y, y^{\prime}\right) \in \partial D \times \bar{B} \times \bar{B} \backslash \mathcal{W}_{\delta_{1}}$. These together with estimate $f(y) f\left(y^{\prime}\right) \geq C^{2}$ for a.e. $y \in B$ gives

$$
\begin{gathered}
J(\tau)=\frac{\tau}{(4 \pi)^{2}} \int_{\mathcal{W}_{\delta_{1}}} d S_{x} d y d y^{\prime}\left(k(x, y)-\frac{1}{\tau} l(x, y)\right) \frac{e^{-\tau\left(|x-y|+\left|x-y^{\prime}\right|\right)}}{|x-y|\left|x-y^{\prime}\right|} f(y) f\left(y^{\prime}\right) \\
+\frac{\tau}{(4 \pi)^{2}} \int_{\partial D \times B \times B \backslash \mathcal{W}_{\delta_{1}}} d S_{x} d y d y^{\prime}\left(k(x, y)-\frac{1}{\tau} l(x, y)\right) \frac{e^{-\tau\left(|x-y|+\left|x-y^{\prime}\right|\right)}}{|x-y|\left|x-y^{\prime}\right|} f(y) f\left(y^{\prime}\right) \\
\geq \frac{\left(C^{\prime} / 2-\tau^{-1} C_{1}\right) \tau C^{2}}{(4 \pi)^{2}(d+\epsilon)^{2}} \int_{\mathcal{W}_{\delta_{1}}} d S_{x} d y d y^{\prime} e^{-\tau\left(|x-y|+\left|x-y^{\prime}\right|\right)}+O\left(\tau e^{-2 \tau d} e^{-\tau \delta_{2}}\right),
\end{gathered}
$$

where

$$
C_{1}=\frac{1}{\operatorname{dist}(D, B)}+\|\beta\|_{L^{\infty}(\partial D)} .
$$

Choose $\left(x_{0}, y_{0}\right) \in \mathcal{M}$. Since $\left|x_{0}-y_{0}\right|=d$, we have

$$
\begin{gathered}
|x-y|+\left|x-y^{\prime}\right| \leq 2\left|x-x_{0}\right|+\left|x_{0}-y\right|+\left|x_{0}-y^{\prime}\right| \\
\leq 2\left|x-x_{0}\right|+2 d+\left|y_{0}-y\right|+\left|y_{0}-y^{\prime}\right| .
\end{gathered}
$$

From the definition of $\mathcal{W}_{\delta_{1}}$ we have

$$
\begin{gathered}
\int_{\mathcal{W}_{\delta_{1}}} d S_{x} d y d y^{\prime} e^{-\tau\left(|x-y|+\left|x-y^{\prime}\right|\right)} \geq \int_{\partial D \cap B_{\delta_{1}}\left(x_{0}\right)} d S_{x} \int_{B \cap B_{\delta_{1}}\left(y_{0}\right)} d y \int_{B \cap B_{\delta_{1}}\left(y_{0}\right)} d y^{\prime} e^{-\tau\left(|x-y|+\left|x-y^{\prime}\right|\right)} \\
\geq e^{-2 \tau d} \int_{\partial D \cap B_{\delta_{1}}\left(x_{0}\right)} e^{-2 \tau\left|x-x_{0}\right|} d S_{x}\left(\int_{B \cap B_{\delta_{1}}\left(y_{0}\right)} e^{-\tau\left|y_{0}-y\right|} d y\right)^{2} .
\end{gathered}
$$

Here we make use of two facts which are essentially same as Claims 3 and 4 in [12]:

for all $\delta>0$ we have

$$
\liminf _{\tau \longrightarrow \infty} \tau^{3} \int_{B \cap B_{\delta}\left(y_{0}\right)} e^{-\tau\left|y_{0}-y\right|} d y>0
$$

and

$$
\liminf _{\tau \longrightarrow \infty} \tau^{2} \int_{\partial D \cap B_{\delta}\left(x_{0}\right)} e^{-2 \tau\left|x-x_{0}\right|} d S_{x}>0 .
$$


From these, (2.37) and (2.38) we see that (2.36) for $\mu=7$ is true.

It follows from (2.26) that

$$
\begin{gathered}
\int_{\mathbf{R}^{3} \backslash \bar{D}}|\nabla \epsilon|^{2} d x+\tau^{2} \int_{\mathbf{R}^{3} \backslash \bar{D}}|\epsilon|^{2} d x+\int_{\partial D} \beta|\epsilon|^{2} d S \\
\geq\left(1-K\|\beta\|_{L^{\infty}(\partial D)} \eta^{2}\right) \int_{\Omega \backslash \bar{D}}|\nabla \epsilon|^{2} d x+\int_{\mathbf{R}^{3} \backslash \bar{\Omega}}|\nabla \epsilon|^{2} d x \\
+\left(\tau^{2}-K\|\beta\|_{L^{\infty}(\partial D)} \eta^{-2}\right) \int_{\Omega \backslash \bar{D}}|\epsilon|^{2} d x+\tau^{2} \int_{\mathbf{R}^{3} \backslash \bar{\Omega}}|\epsilon|^{2} d x \\
\geq\left(\tau^{2}-K\|\beta\|_{L^{\infty}(\partial D)} \eta^{-2}\right) \int_{\mathbf{R}^{3} \backslash \bar{D}}|\epsilon|^{2} d x \\
\geq \frac{\tau^{2}}{2} \int_{\mathbf{R}^{3} \backslash \bar{D}}|\epsilon|^{2} d x
\end{gathered}
$$

for a fixed $\eta$ with $1 \geq K\|\beta\|_{L^{\infty}(\partial D)} \eta^{2}$ and $\tau \geq \sqrt{2 K\|\beta\|_{L^{\infty}(\partial D)}} \eta^{-1}$. Moreover we have

$$
\begin{gathered}
\frac{\tau^{2}}{2} \int_{\mathbf{R}^{3} \backslash \bar{D}}|\epsilon|^{2} d x+e^{-\tau T} \int_{\mathbf{R}^{3} \backslash \bar{D}} F \epsilon d x=\frac{1}{2} \int_{\mathbf{R}^{3} \backslash \bar{D}}\left|\tau \epsilon+\frac{e^{-\tau T}}{\tau} F\right|^{2} d x-\frac{e^{-2 \tau T}}{2 \tau^{2}} \int_{\mathbf{R}^{3} \backslash \bar{D}}|F|^{2} d x \\
\geq-\frac{e^{-2 \tau T}}{2 \tau^{2}} \int_{\mathbf{R}^{3} \backslash \bar{D}}|F|^{2} d x
\end{gathered}
$$

and

$$
\begin{gathered}
\tau \int_{\partial D} \gamma|\epsilon|^{2} d S+e^{-\tau T} \int_{\partial D} G \epsilon d S \\
=\tau \int_{\partial D} \gamma\left|\epsilon+\frac{e^{-\tau T}}{2 \tau} u(x, T)\right|^{2} d S-\frac{e^{-2 \tau T}}{4 \tau} \int_{\partial D} \gamma|u(x, T)|^{2} d x \\
\geq-\frac{e^{-2 \tau T}}{4 \tau} \int_{\partial D} \gamma|u(x, T)|^{2} d x .
\end{gathered}
$$

Note that we have made use of the assumption $\gamma \geq 0$ on $\partial D$. Now it follows from (2.15), (2.39), (2.40) and (2.41) that

$$
\begin{gathered}
I(\tau)=J(\tau)+\int_{\mathbf{R}^{3} \backslash \bar{D}}|\nabla \epsilon|^{2} d x+\tau^{2} \int_{\mathbf{R}^{3} \backslash \bar{D}}|\epsilon|^{2} d x+\int_{\partial D} c(x, \tau)|\epsilon|^{2} d S \\
+e^{-\tau T} \int_{\mathbf{R}^{3} \backslash \bar{D}} F \epsilon d x-e^{-\tau T} \int_{\Omega \backslash \bar{D}} F v d x+e^{-\tau T} \int_{\partial D} G \epsilon d S \\
\geq J(\tau)-\frac{e^{-2 \tau T}}{2 \tau^{2}} \int_{\mathbf{R}^{3} \backslash \bar{D}}|F|^{2} d x-\frac{e^{-2 \tau T}}{4 \tau} \int_{\partial D} \gamma|u(x, T)|^{2} d x-e^{-\tau T} \int_{\Omega \backslash \bar{D}} F v d x \\
=J(\tau)+O\left(e^{-2 \tau T}+\tau e^{-\tau(T+\operatorname{dist}(\Omega, B))}\right) .
\end{gathered}
$$


and thus

$$
\begin{gathered}
\tau^{\mu} e^{2 \tau \operatorname{dist}(D, B)} I(\tau) \geq \tau^{\mu} e^{2 \tau \operatorname{dist}(D, B)} J(\tau) \\
+O\left(\tau^{\mu} e^{-2 \tau(T-\operatorname{dist}(D, B))}+\tau^{\mu+1} e^{-\tau(T-2 \operatorname{dist}(D, B)+\operatorname{dist}(\Omega, B))}\right) .
\end{gathered}
$$

Now from this together with (2.36) we obtain (2.34).

\subsection{Case (A2). An estimate of $I(\tau)$ from above}

In this subsection we prove that there exists a positive constant $\mu$ such that

$$
\liminf _{\tau \longrightarrow \infty}\left(-\tau^{\mu} e^{2 \tau \operatorname{dist}(D, B)} I(\tau)\right)>0
$$

provided $\gamma$ satisfies (A2).

Having (2.16) and (2.42), we immediately obtain (1.6) and (1.7).

The proof of (2.42) starts with rewriting (2.12) as

$$
\begin{gathered}
\int_{\partial D} \frac{1}{4 c(x, \tau)}\left|\left(\frac{\partial v}{\partial \nu}-c(x, \tau) v\right)-e^{-\tau T} G\right|^{2} d S+\frac{e^{-2 \tau T}}{4 \tau^{2}} \int_{\mathbf{R}^{3} \backslash \bar{D}}|F|^{2} d x \\
=\int_{\partial D} c(x, \tau)\left|\epsilon-\frac{1}{2 c(x, \tau)}\left\{\left(\frac{\partial v}{\partial \nu}-c(x, \tau) v\right)-e^{-\tau T} G\right\}\right|^{2} d S \\
+\int_{\mathbf{R}^{3} \backslash \bar{D}}\left(|\nabla \epsilon|^{2}+\tau^{2}\left|\epsilon+\frac{e^{-\tau T}}{2 \tau^{2}} F\right|^{2}\right) d x
\end{gathered}
$$

Since

$$
\left|\epsilon+\frac{e^{-\tau T}}{2 \tau^{2}} F\right|^{2} \geq \frac{1}{2}|\epsilon|^{2}-\frac{e^{-2 \tau T}}{4 \tau^{4}}|F|^{2}
$$

and

$$
\begin{gathered}
\left|\epsilon-\frac{1}{2 c(x, \tau)}\left\{\left(\frac{\partial v}{\partial \nu}-c(x, \tau) v\right)-e^{-\tau T} G\right\}\right|^{2} \\
\geq \frac{1}{2}|\epsilon|^{2}-\frac{1}{4 c(x, \tau)^{2}}\left|\left(\frac{\partial v}{\partial \nu}-c(x, \tau) v\right)-e^{-\tau T} G\right|^{2},
\end{gathered}
$$

it follows from (2.43) that

$$
\begin{gathered}
\int_{\partial D} \frac{c(x, \tau)}{2}|\epsilon|^{2} d S+\int_{\mathbf{R}^{3} \backslash \bar{D}}\left(|\nabla \epsilon|^{2}+\frac{\tau^{2}}{2}|\epsilon|^{2}\right) d x \\
\leq \int_{\partial D} \frac{1}{2 c(x, \tau)}\left|\left(\frac{\partial v}{\partial \nu}-c(x, \tau) v\right)-e^{-\tau T} G\right|^{2} d S+\frac{e^{-2 \tau T}}{2 \tau^{2}} \int_{\mathbf{R}^{3} \backslash \bar{D}}|F|^{2} d x
\end{gathered}
$$


and thus

$$
\begin{gathered}
\int_{\partial D} c(x, \tau)|\epsilon|^{2} d S+\int_{\mathbf{R}^{3} \backslash \bar{D}}\left(|\nabla \epsilon|^{2}+\tau^{2}|\epsilon|^{2}\right) d x \\
\leq \int_{\partial D} \frac{1}{c(x, \tau)}\left|\left(\frac{\partial v}{\partial \nu}-c(x, \tau) v\right)-e^{-\tau T} G\right|^{2} d S+\frac{e^{-2 \tau T}}{\tau^{2}} \int_{\mathbf{R}^{3} \backslash \bar{D}}|F|^{2} d x \\
=\int_{\partial D} \frac{1}{c(x, \tau)}\left|\frac{\partial v}{\partial \nu}-c(x, \tau) v\right|^{2} d S+O\left(e^{-\tau(T+\operatorname{dist}(D, B))}+e^{-2 \tau T}\right) .
\end{gathered}
$$

It follows from (2.17) that

$$
e^{-\tau T} \int_{\mathbf{R}^{3} \backslash \bar{D}} F \epsilon d x=O\left(\tau\left(e^{-2 \tau T}+e^{-\tau(T+\operatorname{dist}(D, B))}\right)\right)
$$

and from (2.28) that

$$
e^{-\tau T} \int_{\partial D} G \epsilon d S=O\left(\tau^{1 / 2}\left(e^{-2 \tau T}+e^{-\tau(T+\operatorname{dist}(D, B))}\right) .\right.
$$

Now applying $(2.31),(2.32),(2.44),(2.45)$ and $(2.46)$ to $(2.15)$ we obtain

$$
I(\tau) \leq \tilde{J}(\tau)+O\left(\tau\left(e^{-2 \tau T}+e^{-\tau(T+\operatorname{dist}(D, B))}\right)\right)+O\left(\tau e^{-\tau(T+\operatorname{dist}(\Omega, B))}\right),
$$

where

$$
\tilde{J}(\tau)=J(\tau)+\int_{\partial D} \frac{1}{c(x, \tau)}\left|\frac{\partial v}{\partial \nu}-c(x, \tau) v\right|^{2} d S .
$$

From (2.35) one gets

$$
\begin{gathered}
\tilde{J}(\tau)=\int_{\partial D} \frac{1}{c(x, \tau)}\left(\frac{\partial v}{\partial \nu}-c(x, \tau) v\right) \frac{\partial v}{\partial \nu} d S \\
=\left(\frac{\tau}{4 \pi}\right)^{2} \int_{\partial D} \frac{1}{c(x, \tau)} d S_{x} \\
\times \int_{B \times B}\left(k\left(x, y^{\prime}\right)-\frac{1}{\tau} l\left(x, y^{\prime}\right)\right)\left(\tilde{k}(x, y)-\frac{1}{\tau} \tilde{l}(x, y)\right) \frac{e^{-\tau\left(\left|x-y^{\prime}\right|+|x-y|\right)}}{\left|x-y^{\prime}\right||x-y|} f\left(y^{\prime}\right) f(y) d y^{\prime} d y
\end{gathered}
$$

where $k$ is the same as that of Lemma 2.2 and

$$
\begin{aligned}
& \tilde{k}(x, y)=\frac{(y-x) \cdot \nu(x)}{|x-y|}, \\
& \tilde{l}(x, y)=\frac{(y-x) \cdot \nu(x)}{|x-y|^{2}} .
\end{aligned}
$$

From (A2) we have $k\left(x, y^{\prime}\right) \leq-C^{\prime}$ and thus

$$
k\left(x, y^{\prime}\right)-\frac{1}{\tau} l\left(x, y^{\prime}\right) \leq-\frac{C^{\prime}}{2}, \quad\left(x, y^{\prime}\right) \in \partial D \times B
$$


for all $\tau \geq \tau_{0}$ with $\tau_{0}>>1$. Now give $\epsilon=1 / 2$ in Claim I of Lemma 2.2 and choose $\delta_{1}$ and corresponding $\delta_{2}$ in Claim 2. It is easy to see that one can apply the same argument as done in Lemma 2.2 to $\tilde{J}(\tau)$ where $\tilde{k}(x, y)-(1 / \tau) \tilde{l}(x, y)$ plays the same role as $k(x, y)-(1 / \tau) l(x, y)$ in Lemma 2.2. Thus we conclude that there exists $\mu>0$ such that

$$
\liminf _{\tau \longrightarrow \infty}\left\{-\tau^{\mu} e^{2 \tau \operatorname{dist}(D, B)} \tilde{J}(\tau)\right\}>0
$$

and thus from (2.47) one gets (2.42).

\subsection{Proof of Theorem 1.2}

Since $\bar{B} \cap \bar{D}=\emptyset$, one can find a bounded open set $\Omega$ with a smooth boundary such that $\mathbf{R}^{3} \backslash \bar{\Omega}$ is connected, $\bar{D} \subset \Omega$ and $\bar{B} \cap \bar{\Omega}=\emptyset$. We consider $I(\tau)$ for this $\Omega$.

Since supp $f \cap \bar{\Omega}=\emptyset$, it follows from (2.3) that

$$
I(\tau)=\int_{\partial D} \frac{\partial v}{\partial \nu} w d S-\int_{\partial D} c(x, \tau) w v d S-e^{-\tau T} \int_{\partial D} G v d S-e^{-\tau T} \int_{\Omega \backslash \bar{D}} F v d x
$$

Substituting $\phi=w$ into $(2.10)$, we have

$$
\int_{\partial D} \frac{\partial v}{\partial \nu} w d S=\int_{\mathbf{R}^{3} \backslash \bar{D}} f w d x-\int_{\mathbf{R}^{3} \backslash \bar{D}}\left(\nabla v \cdot \nabla w+\tau^{2} v w\right) d x
$$

and thus (2.48) becomes

$$
\begin{gathered}
I(\tau)=\int_{\mathbf{R}^{3} \backslash \bar{D}} f(w-v) d x-\left\{\int_{\mathbf{R}^{3} \backslash \bar{D}}\left(\nabla w \cdot \nabla v+\left(\tau^{2} w-f\right) v\right) d x+\int_{\partial D} c(x, \tau) w v d S\right\} \\
-e^{-\tau T} \int_{\partial D} G v d S-e^{-\tau T} \int_{\Omega \backslash \bar{D}} F v d x .
\end{gathered}
$$

Now it follows from this and (2.2) for $\phi=\left.v\right|_{\mathbf{R}^{3} \backslash \bar{D}}$ that

$$
I(\tau)=\int_{\mathbf{R}^{3} \backslash \bar{D}} f(w-v) d x+e^{-\tau T} \int_{\mathbf{R}^{3} \backslash \bar{\Omega}} F v d x .
$$

Since $\|F\|_{L^{2}\left(\mathbf{R}^{3} \backslash \bar{D}\right)}=O(\tau)$ and

$$
\|v\|_{L^{2}\left(\mathbf{R}^{3}\right)} \leq \tau^{-2}\|f\|_{L^{2}\left(\mathbf{R}^{3}\right)}
$$

(2.49) yields

$$
\int_{\mathbf{R}^{3} \backslash \bar{D}} f(w-v) d x=I(\tau)+O\left(\tau^{-1} e^{-\tau T}\right) .
$$

Now (1.10) and (1.12) follow from (2.51) together with (2.16) and (2.34) in case (A1); (1.11) and (1.12) follow from (2.51) together with (2.16) and (2.42) in case (A2). 


\section{Proof of Theorems 1.3 and 1.4}

Set $V=H^{1}\left(\mathbf{R}^{3}\right)$ and $H=L^{2}\left(\mathbf{R}^{3}\right)$. Applying Theorem 1 on p 558 in [1] to (1.13), we know that: given $u^{0} \in V$ and $u^{1} \in H$ there exists a unique $u$ satisfying

$$
u \in L^{2}(0, T ; V), u^{\prime} \in L^{2}(0, T ; V), d / d t\left(C\left(u^{\prime}(\cdot)\right) \in L^{2}\left(0, T ; V^{\prime}\right)\right.
$$

such that, for all $\phi \in V$

$$
\left.<\frac{d}{d t} C\left(u^{\prime}(t)\right), \phi>+a(u(t), \phi)=0, \text { a.e. } t \in\right] 0, T[
$$

and $u(0)=u^{0}$ and $u^{\prime}(0)=u^{1}$, where

$$
a(u, v)=\int_{\mathbf{R}^{3}} \nabla u \cdot \nabla v d x, u, v \in V
$$

and $C: H \longrightarrow H$ is the bounded linear operator defined by

$$
C(u)=\alpha u, u \in H \text {. }
$$

Note that this $C$ satisfies (5.11) on p 553 in [1] under the condition $\alpha(x) \geq C$ a.e. $x \in \mathbf{R}^{3}$ for some positive constant. Note that at this step we do not make use of condition $\alpha(x)=1$ a.e. $x \in \mathbf{R}^{3} \backslash D$.

Note also that this is the case when $b=0$ in Theorem 1 . However, the equation is homogeneous, that is, $f=0$ in their notation and by virtue of this their proof covers also this case.

In this section we say that this $u$ for $u^{0}=0$ and $u^{1}=f$ is the weak solution of (1.13).

We see that $w$ given by (1.14) belongs to $V$ and applying integration by parts to (3.1) multiplied by $e^{-\tau T}$, we obtain, for all $\phi \in V$

$$
\int_{\mathbf{R}^{3}}\left(\nabla w \cdot \nabla \phi+\tau^{2} \alpha w \phi\right) d x-\int_{\mathbf{R}^{3}} \alpha f \phi d x+\int_{\mathbf{R}^{3}} \boldsymbol{F} \phi d x=0,
$$

where

$$
\boldsymbol{F}(x)=e^{-\tau T} \alpha(x)\left(u^{\prime}(x, T)+\tau u(x, T)\right) .
$$

This means that $w$ is the weak solution of the following equation

$$
\left(\triangle-\alpha \tau^{2}\right) w+\alpha f=\boldsymbol{F} \text { in } \mathbf{R}^{3} .
$$

By the same reason as $w$ in Section 2 we have $w \in H_{\text {loc }}^{2}\left(\mathbf{R}^{3}\right)$ and (3.3) holds a.e. $x \in \mathbf{R}^{3}$.

Since $v$ satisfies (1.3) in the weak sense, we see that $w-v$ satisfies, in a weak sense,

$$
\left(\triangle-\alpha \tau^{2}\right)(w-v)=(\alpha-1)\left(\tau^{2} v-f\right)+\boldsymbol{F} \text { in } \mathbf{R}^{3} .
$$

\subsection{Two expressions}

We start with the following two key expressions. Note that in the proposition we do not assume that $\operatorname{supp} f \cap \bar{\Omega}=\emptyset$. 
Proposition 3.1. We have:

$$
\begin{gathered}
\int_{\partial \Omega}\left(\frac{\partial v}{\partial \nu} w-\frac{\partial w}{\partial \nu} v\right) d S \\
=\int_{\mathbf{R}^{3}}\left\{|\nabla(w-v)|^{2}+\tau^{2} \alpha|w-v|^{2}\right\} d x+\tau^{2} \int_{D}(1-\alpha)|v|^{2} d x \\
+\int_{\mathbf{R}^{3}}(1-\alpha) f(w-v) d x+\int_{\mathbf{R}^{3}} \boldsymbol{F}(w-v) d x+\int_{\Omega} f(\alpha v-w) d x-\int_{\Omega} \boldsymbol{F} v d x \\
\int_{\partial \Omega}\left(\frac{\partial w}{\partial \nu} v-\frac{\partial v}{\partial \nu} w\right) d S \\
=\int_{\mathbf{R}^{3}}\left\{|\nabla(v-w)|^{2}+\tau^{2}|v-w|^{2}\right\} d x+\tau^{2} \int_{\Omega}(\alpha-1)|w|^{2} d x \\
+\int_{\mathbf{R}^{3}}(\alpha-1) f(v-w) d x-\int_{\mathbf{R}^{3}} \boldsymbol{F}(v-w) d x+\int_{\Omega} f(w-\alpha v) d x+\int_{\Omega} \boldsymbol{F} v d x .
\end{gathered}
$$

Proof. The proof of (3.5) and (3.6) are rather simpler than that of (2.3) and (2.4). First we give a proof of (3.5). Since $v \in H^{2}(\Omega)$ and satisfies $\left(\triangle-\tau^{2}\right) v+f=0$ a.e. $x \in \Omega$, integration by parts gives

$$
\int_{\partial \Omega} \frac{\partial v}{\partial \nu} w d S=\int_{\Omega}\left(\tau^{2} v-f\right) w d x+\int_{\Omega} \nabla v \cdot \nabla w d x
$$

and by the same reason for $w$ we have

$$
\int_{\partial \Omega} \frac{\partial w}{\partial \nu} v d S=\int_{\Omega} \nabla w \cdot \nabla v d x+\tau^{2} \int_{\Omega} \alpha w v d x-\int_{\Omega} \alpha f v d x+\int_{\Omega} \boldsymbol{F} v d x .
$$

These yield

$$
\int_{\partial \Omega}\left(\frac{\partial v}{\partial \nu} w-\frac{\partial w}{\partial \nu} v\right) d S=\tau^{2} \int_{\Omega}(1-\alpha) w v d x+\int_{\Omega} f(\alpha v-w) d x-\int_{\Omega} \boldsymbol{F} v d x
$$

Write the integral in the first term of the right hand-side as

$$
\int_{\Omega}(1-\alpha) w v d x=\int_{\Omega}(1-\alpha) v(w-v) d x+\int_{\Omega}(1-\alpha)|v|^{2} d x .
$$

On the other hand, from the weak form of (3.4) and integration by parts we have

$$
\begin{gathered}
-\int_{\mathbf{R}^{3}}|\nabla(w-v)|^{2} d x-\tau^{2} \int_{\mathbf{R}^{3}} \alpha|w-v|^{2} d x \\
=\tau^{2} \int_{\mathbf{R}^{3}}(\alpha-1) v(w-v) d x-\int_{\mathbf{R}^{3}}(\alpha-1) f(w-v) d x+\int_{\mathbf{R}^{3}} \boldsymbol{F}(w-v) d x,
\end{gathered}
$$

that is,

$$
\begin{gathered}
\tau^{2} \int_{\mathbf{R}^{3}}(1-\alpha) v(w-v) d x \\
=\int_{\mathbf{R}^{3}}\left\{|\nabla(w-v)|^{2}+\tau^{2} \alpha|w-v|^{2}\right\} d x+\int_{\mathbf{R}^{3}}(1-\alpha) f(w-v) d x+\int_{\mathbf{R}^{3}} \boldsymbol{F}(w-v) d x .
\end{gathered}
$$


Since $1-\alpha(x)=0$ outside $D$, from (3.7), (3.8) and (3.9) we obtain the desired expression.

Second we give a proof of (3.6).

We change the role of $v$ and $w$. Introducing

$$
\tilde{f}=f-\frac{1}{\alpha} \boldsymbol{F}, \tilde{\boldsymbol{F}}=-\frac{1}{\alpha} \boldsymbol{F}
$$

one can rewrite equations (1.4) and (3.3) as

$$
\left(\triangle-\tau^{2}\right) v+\tilde{f}=\tilde{\boldsymbol{F}} \text { in } \mathbf{R}^{3}
$$

and

$$
\left(\triangle-\alpha \tau^{2}\right) w+\alpha \tilde{f}=0 \text { in } \mathbf{R}^{3}
$$

Then $v-w$ satisfies

$$
\begin{gathered}
\left(\triangle-\tau^{2}\right)(v-w)=\tilde{\boldsymbol{F}}-\tilde{f}-\left(\triangle-\tau^{2}\right) w \\
=\tilde{\boldsymbol{F}}-\tilde{f}-\left\{\triangle-\alpha \tau^{2}+(\alpha-1) \tau^{2}\right\} w \\
=\tilde{\boldsymbol{F}}-\tilde{f}+\alpha \tilde{f}+(1-\alpha) \tau^{2} w \\
=(1-\alpha)\left(\tau^{2} w-\tilde{f}\right)+\tilde{\boldsymbol{F}} .
\end{gathered}
$$

Integration by parts gives

$$
\begin{gathered}
\int_{\partial \Omega} \frac{\partial w}{\partial \nu} v d S=\int_{\Omega}(\triangle w) v d x+\int_{\Omega} \nabla w \cdot \nabla v d x \\
=\int_{\Omega} \alpha \tau^{2} w v d x-\int_{\Omega} \alpha \tilde{f} v d x+\int_{\Omega} \nabla w \cdot \nabla v d x \\
=\int_{\Omega} \alpha\left(\tau^{2} w-\tilde{f}\right) v d x+\int_{\Omega} \nabla w \cdot \nabla v d x
\end{gathered}
$$

and

$$
\begin{gathered}
\int_{\partial \Omega} \frac{\partial v}{\partial \nu} w d S=\int_{\Omega}(\triangle v) w d x+\int_{\Omega} \nabla v \cdot \nabla w d x \\
=\int_{\Omega}\left(\tau^{2} v-\tilde{f}+\tilde{\boldsymbol{F}}\right) w d x+\int_{\Omega} \nabla v \cdot \nabla w d x \\
=\int_{\Omega} \nabla v \cdot \nabla w d x+\tau^{2} \int_{\Omega} v w d x-\int_{\Omega} \tilde{f} w d x+\int_{\Omega} \tilde{\boldsymbol{F}} w d x .
\end{gathered}
$$

From these we obtain

$$
\begin{gathered}
\int_{\partial \Omega}\left(\frac{\partial w}{\partial \nu} v-\frac{\partial v}{\partial \nu} w\right) d S \\
=\tau^{2} \int_{\Omega}(\alpha-1) w v d x+\int_{\Omega} \tilde{f}(w-\alpha v) d x-\int_{\Omega} \tilde{\boldsymbol{F}} w d x .
\end{gathered}
$$


Here rewrite the integral of the first term in the right-hand side:

$$
\int_{\Omega}(\alpha-1) w v d x=\int_{\Omega}(\alpha-1) w(v-w) d x+\int_{\Omega}(\alpha-1)|w|^{2} d x .
$$

It follows from the weak form of (3.10) that

$$
\begin{gathered}
-\int_{\mathbf{R}^{3}}|\nabla(v-w)|^{2} d x-\tau^{2} \int_{\mathbf{R}^{3}}|v-w|^{2} d x \\
=\int_{\mathbf{R}^{3}}(1-\alpha)\left(\tau^{2} w-\tilde{f}\right)(v-w) d x+\int_{\mathbf{R}^{3}} \tilde{\boldsymbol{F}}(v-w) d x .
\end{gathered}
$$

This yields

$$
\begin{gathered}
\tau^{2} \int_{\mathbf{R}^{3}}(\alpha-1) w(v-w) d x \\
=\int_{\mathbf{R}^{3}}\left\{|\nabla(v-w)|^{2}+\tau^{2}|v-w|^{2}\right\} d x-\int_{\mathbf{R}^{3}}(1-\alpha) \tilde{f}(v-w) d x+\int_{\mathbf{R}^{3}} \tilde{\boldsymbol{F}}(v-w) d x \\
=\int_{\mathbf{R}^{3}}\left\{|\nabla(v-w)|^{2}+\tau^{2}|v-w|^{2}\right\} d x+\int_{\mathbf{R}^{3}}\{\tilde{\boldsymbol{F}}+(\alpha-1) \tilde{f}\}(v-w) d x .
\end{gathered}
$$

Now from (3.11), (3.12) and (3.13) we obtain

$$
\begin{gathered}
\int_{\partial \Omega}\left(\frac{\partial w}{\partial \nu} v-\frac{\partial v}{\partial \nu} w\right) d S \\
=\tau^{2} \int_{\Omega}(\alpha-1)|w|^{2} d x+\int_{\mathbf{R}^{3}}\left\{|\nabla(v-w)|^{2}+\tau^{2}|v-w|^{2}\right\} d x \\
+\int_{\mathbf{R}^{3}}\{\tilde{\boldsymbol{F}}+(\alpha-1) \tilde{f}\}(v-w) d x+\int_{\Omega} \tilde{f}(w-\alpha v) d x-\int_{\Omega} \tilde{\boldsymbol{F}} w d x .
\end{gathered}
$$

Since

$$
\begin{gathered}
\tilde{\boldsymbol{F}}+(\alpha-1) \tilde{f}=-\boldsymbol{F}+(\alpha-1) f, \\
\tilde{f}-\tilde{\boldsymbol{F}}=f
\end{gathered}
$$

this is nothing but (3.6).

Now assume that supp $f \cap \bar{\Omega}=\emptyset$. Set

$$
K(\tau)=\int_{\partial \Omega}\left(\frac{\partial v}{\partial \nu} w-\frac{\partial w}{\partial \nu} v\right) d S, \tau>0 .
$$

Since $\alpha(x)=1$ a. e. $x \in \mathbf{R}^{3} \backslash D,(3.5)$ and (3.6) become the following two expressions of $K(\tau)$ :

$$
\begin{gathered}
K(\tau)=\int_{\mathbf{R}^{3}}\left\{|\nabla(w-v)|^{2}+\tau^{2} \alpha|w-v|^{2}\right\} d x \\
+\tau^{2} \int_{D}(1-\alpha)|v|^{2} d x+\int_{\mathbf{R}^{3}} \boldsymbol{F}(w-v) d x-\int_{\Omega} \boldsymbol{F} v d x
\end{gathered}
$$




$$
\begin{gathered}
-K(\tau)=\int_{\mathbf{R}^{3}}\left\{|\nabla(v-w)|^{2}+\tau^{2}|v-w|^{2}\right\} d x+\tau^{2} \int_{\Omega}(\alpha-1)|w|^{2} d x \\
-\int_{\mathbf{R}^{3}} \boldsymbol{F}(v-w) d x+\int_{\Omega} \boldsymbol{F} v d x .
\end{gathered}
$$

\subsection{An estimate of $|K(\tau)|$ from above}

We describe the proof of the following estimate as $\tau \longrightarrow \infty$ :

$$
e^{2 \tau \operatorname{dist}(D, B)}|K(\tau)|=O\left(\tau^{2}\right) .
$$

Since $(\alpha(x)-1) f(x)=0$ for a.e. $x \in \mathbf{R}^{3}$ it follows from (3.9) that

$$
\begin{gathered}
\int_{\mathbf{R}^{3}}\left\{|\nabla(w-v)|^{2}+\tau^{2} \alpha|w-v|^{2}\right\} d x \\
=\tau^{2} \int_{D}(1-\alpha) v(w-v) d x-\int_{\mathbf{R}^{3}} \boldsymbol{F}(w-v) d x .
\end{gathered}
$$

Applying a much simpler argument than that for the derivation of (2.17) and (2.18) in Lemma 2.1 to (3.17) and using the estimate

$$
\|\boldsymbol{F}\|_{L^{2}\left(\mathbf{R}^{3}\right)}^{2}=O\left(\tau^{2} e^{-2 \tau T}\right),
$$

we obtain, as $\tau \longrightarrow \infty$

$$
\begin{gathered}
\|w-v\|_{L^{2}\left(\mathbf{R}^{3}\right)}^{2}=O\left(e^{-2 \tau T}+e^{-2 \tau \operatorname{dist}(D, B)}\right), \\
\|\nabla(w-v)\|_{L^{2}\left(\mathbf{R}^{3}\right)}^{2}=O\left(\tau^{2}\left(e^{-2 \tau T}+e^{-2 \tau \operatorname{dist}(D, B)}\right)\right)
\end{gathered}
$$

and thus one gets

$$
\left|\int_{\mathbf{R}^{3}} \boldsymbol{F}(w-v) d x\right|=O\left(\tau\left(e^{-2 \tau T}+e^{-\tau(T+\operatorname{dist}(D, B))}\right)\right) .
$$

Since $\|v\|_{L^{2}(\Omega)}=O\left(e^{-\tau \operatorname{dist}(\Omega, B)}\right)$, from (3.18) one gets also

$$
\left|\int_{\Omega} \boldsymbol{F} v d x\right|=O\left(\tau e^{-\tau(T+\operatorname{dist}(\Omega, B))}\right) .
$$

Now it follows from (3.14), $\|v\|_{L^{2}(D)}=O\left(e^{-\tau \operatorname{dist}(D, B)}\right)$ and (3.18) to (3.21) that, as $\tau \longrightarrow \infty$

$$
\left.|K(\tau)|=O\left(\tau^{2}\left(e^{-2 \tau T}+e^{-2 \tau \operatorname{dist}(D, B)}\right)\right)+\tau e^{-\tau(T+\operatorname{dist}(\Omega, B))}\right) .
$$

From this we obtain (3.16). 


\subsection{Case (B1). An estimate of $K(\tau)$ from below}

In this subsection we consider the case when $\alpha(x) \leq 1-C^{\prime}$ a.e. $x \in D$ and prove that:

$$
\liminf _{\tau \longrightarrow \infty} \tau^{4} e^{2 \tau \operatorname{dist}(D, B)} K(\tau)>0 .
$$

Using the completing square

$$
\tau^{2} \alpha|w-v|^{2}+\boldsymbol{F}(w-v)=\tau^{2} \alpha\left|(w-v)+\frac{\boldsymbol{F}}{2 \tau^{2} \alpha}\right|^{2}-\frac{|\boldsymbol{F}|^{2}}{4 \tau^{2} \alpha},
$$

from (3.14), (3.18) and (3.21) we obtain

$$
K(\tau) \geq C^{\prime} \tau^{2} \int_{D}|v|^{2} d x+O\left(e^{-2 \tau T}+\tau e^{-\tau(T+\operatorname{dist}(\Omega, B))}\right) .
$$

Here we cite the following fact established in [9]:

$$
\liminf _{\tau \longrightarrow \infty} \tau^{6} e^{2 \tau \operatorname{dist}(D, B)} \int_{D}|v|^{2} d x>0 .
$$

This together with (3.23) yields (3.22).

\subsection{Case (B2). An estimate of $K(\tau)$ from above}

Next consider the case when $\alpha(x) \geq 1+C^{\prime}$ a.e. $x \in D$. In this subsection we prove the following estimate:

$$
\liminf _{\tau \longrightarrow \infty}\left(-\tau^{4} e^{2 \tau \operatorname{dist}(D, B)} K(\tau)\right)>0 .
$$

We make use of the following completing the square:

$$
\begin{gathered}
|v-w|^{2}+(\alpha-1)|w|^{2}=|v|^{2}-2 v w+\alpha|w|^{2} \\
=\left|\sqrt{\alpha} w-\frac{v}{\sqrt{\alpha}}\right|^{2}+\frac{\alpha-1}{\alpha}|v|^{2} .
\end{gathered}
$$

This together with (3.15), (3.20) and (3.21) gives

$$
\begin{gathered}
-K(\tau) \geq \tau^{2} \int_{D} \frac{\alpha-1}{\alpha}|v|^{2} d x-\int_{\mathbf{R}^{3}} \boldsymbol{F}(v-w) d x+\int_{\Omega} \boldsymbol{F} v d x \\
\geq \frac{C^{\prime} \tau^{2}}{\|\alpha\|_{L^{\infty}\left(\mathbf{R}^{3}\right)}} \int_{D}|v|^{2} d x+O\left(\tau\left(e^{-2 \tau T}+e^{-\tau(T+\operatorname{dist}(D, B))}\right)\right)+O\left(\tau e^{-\tau(T+\operatorname{dist}(\Omega, B))}\right) .
\end{gathered}
$$

Now a combination of this and (3.24) yields (3.25).

It is easy to see that $(1.15) /(1.16)$ and (1.17) follow from the combination of $(3.16)$ and $(3.22) /(3.25)$ in the case when $(\mathrm{B} 1) /(\mathrm{B} 2)$ is satisfied. 


\subsection{Proof of Theorem 1.4.}

Since $\bar{B} \cap \bar{D}=\emptyset$, one can find a bounded open set $\Omega$ with a smooth boundary such that $\mathbf{R}^{3} \backslash \bar{\Omega}$ is connected, $\bar{D} \subset \Omega$ and $\bar{B} \cap \bar{\Omega}=\emptyset$.

We make use of (3.7). Since supp $f \cap \bar{\Omega}=\emptyset,(3.7)$ becomes

$$
\int_{\partial \Omega}\left(\frac{\partial v}{\partial \nu} w-\frac{\partial w}{\partial \nu} v\right) d S=\tau^{2} \int_{\Omega}(1-\alpha) w v d x-\int_{\Omega} \boldsymbol{F} v d x
$$

One can rewrite the first term of this right-hand side as

$$
\begin{gathered}
\tau^{2} \int_{\Omega}(1-\alpha) w v d x=\tau^{2} \int_{\mathbf{R}^{3}}(1-\alpha) w v d x \\
=\tau^{2} \int_{\mathbf{R}^{3}} v w d x-\tau^{2} \int_{\mathbf{R}^{3}} \alpha w v d x \\
=-\int_{\mathbf{R}^{3}} \nabla v \cdot \nabla w d x+\int_{\mathbf{R}^{3}} f w d x-\tau^{2} \int_{\mathbf{R}^{3}} \alpha w v d x \\
=-\int_{\mathbf{R}^{3}}\left(\nabla w \cdot \nabla v+\tau^{2} \alpha w v\right) d x+\int_{\mathbf{R}^{3}} f w d x \\
=-\left(\int_{\mathbf{R}^{3}} \alpha f v d x-\int_{\mathbf{R}^{3}} \boldsymbol{F} v d x\right)+\int_{\mathbf{R}^{3}} f w d x \\
=\int_{\mathbf{R}^{3}} f(w-\alpha v) d x+\int_{\mathbf{R}^{3}} \boldsymbol{F} v d x \\
=\int_{\mathbf{R}^{3}} f(w-v) d x+\int_{\mathbf{R}^{3}}(1-\alpha) f v d x+\int_{\mathbf{R}^{3}} \boldsymbol{F} v d x .
\end{gathered}
$$

Since $(1-\alpha) f=0$ in $\mathbf{R}^{3}$, from this and (3.26) we obtain

$$
\int_{\partial \Omega}\left(\frac{\partial v}{\partial \nu} w-\frac{\partial w}{\partial \nu} v\right) d S=\int_{\mathbf{R}^{3}} f(w-v) d x+\int_{\mathbf{R}^{3} \backslash \bar{\Omega}} \boldsymbol{F} v d x
$$

This together with (2.50) and (3.18) gives

$$
\int_{\mathbf{R}^{3}} f(w-v) d x=\int_{\partial \Omega}\left(\frac{\partial v}{\partial \nu} w-\frac{\partial w}{\partial \nu} v\right) d S+O\left(\tau^{-1} e^{-\tau T}\right) .
$$

Now $(1.18) /(1.19)$ and (1.20) follows from this together with $(3.16)$ and $(3.22) /(3.25)$ in the case $(\mathrm{B} 1) /(\mathrm{B} 2)$.

\section{Remarks and open problems}

\subsection{One space dimensional case}

Let us consider the meaning of conditions (A1) and (A2) in one space dimensional case. 
Let $0<T<\infty$ and $a>0$. Given $f \in L^{2}(\mathbf{R})$ with $\left.\operatorname{supp} f \subset\right]-\infty, a\left[\right.$ let $u=u_{f}(x, t)$ be the weak solution of the following initial boundary value problem:

$$
\begin{gathered}
\left.\partial_{t}^{2} u-\partial_{x}^{2} u=0 \text { in }\right]-\infty, a[\times] 0, T[, \\
\left.u(x, 0)=0, \partial_{t} u(x, 0)=f(x) \text { in }\right]-\infty, a[, \\
-\partial_{x} u(a, t)-\gamma \partial_{t} u(a, t)-\beta u(a, t)=0,0<t<T,
\end{gathered}
$$

where $\gamma$ and $\beta \in \mathbf{R}$ are constant and $\gamma \geq 0$. This is the case when $D=] a, \infty[$. We take $\Omega=] 0, \infty[$ and thus $\partial \Omega=\{0\}$.

Hereafter we choose initial data $f$ in such a way that $\operatorname{supp} f \subset]-\infty, 0[$.

Define

$$
\left.w=w_{f}(x, \tau)=\int_{0}^{T} e^{-\tau t} u_{f}(x, t) d t, x \in\right]-\infty, a[, \tau>0 .
$$

$w$ satisfies

$$
\left.w^{\prime \prime}-\tau^{2} w+f(x)=e^{-\tau T} F(x, \tau) \text { in }\right]-\infty, a[
$$

and

$$
w^{\prime}(a)+c(\tau) w(a)+e^{-\tau T} g(\tau)=0,
$$

where

$$
\begin{gathered}
F(x, \tau)=\partial_{t} u(x, T)+\tau u(x, T), \\
g(\tau)=\gamma u(a, T), \\
c(\tau)=\gamma \tau+\beta .
\end{gathered}
$$

Assume that the support of $f$ is compact and satisfies

$$
\operatorname{supp} f \subset[b-\epsilon, b] \equiv B
$$

with $b<0$ and $\epsilon>0$.

Note that $f \in L^{2}(\mathbf{R})$ with $\left.\left.\operatorname{supp} f \subset\right]-\infty, b\right]$ always satisfies

$$
\left|\int_{b-\epsilon}^{b} e^{-\tau(b-y)} f(y) d y\right|=O\left(\tau^{-1 / 2}\right) .
$$

Now define the indicator function by

$$
I(\tau)=-v^{\prime}(0, \tau) w(0, \tau)+w^{\prime}(0, \tau) v(0, \tau), \tau>0,
$$

where

$$
v=v(x, \tau)=\frac{1}{2 \tau} \int_{-\infty}^{\infty} e^{-\tau|x-y|} f(y) d y .
$$

$v$ belongs to $H^{1}(\mathbf{R})$ and satisfies the equation $v^{\prime \prime}-\tau^{2} v+f(x)=0$ in $\mathbf{R}$.

We have the following asymptotic formula $I(\tau)$ as $\tau \longrightarrow \infty$.

Lemma 4.1. Let $f$ satisfy (4.5). As $\tau \longrightarrow \infty$ we have

$$
\begin{aligned}
I(\tau)=- & \frac{1}{2 \tau} \frac{(\gamma-1) \tau+\beta}{(\gamma+1) \tau+\beta} e^{-2 \tau} \operatorname{dist}(D, B)\left|\int_{b-\epsilon}^{b} e^{-\tau(b-y)} f(y) d y\right|^{2} \\
& +O\left(e^{-\tau(T+\operatorname{dist}(D, B))}+\tau e^{-\tau(T+\operatorname{dist}(\Omega, B))}\right) .
\end{aligned}
$$


Proof. Using one space-dimensional version of (2.14), (2.31) and (2.32), we have

$$
I(\tau)=-\left(v^{\prime}(a)+c(\tau) v(a)\right) w(a)+O\left(e^{-\tau(T+\operatorname{dist}(D, B))}+\tau e^{-\tau(T+\operatorname{dist}(\Omega, B))}\right) .
$$

Note that dist $(D, B)=a-b$ and $\operatorname{dist}(\Omega, B)=-b$.

Since

$$
v^{\prime}(x)=-\frac{1}{2}\left(\int_{-\infty}^{x} e^{-\tau(x-y)} f(y) d y-\int_{x}^{\infty} e^{-\tau(y-x)} f(y) d y\right),
$$

this together with (4.5) and (4.7) gives

$$
\begin{aligned}
v(a) & =\frac{1}{2 \tau} \int_{b-\epsilon}^{b} e^{-\tau(a-y)} f(y) d y, \\
v^{\prime}(a) & =-\frac{1}{2} \int_{b-\epsilon}^{b} e^{-\tau(a-y)} f(y) d y
\end{aligned}
$$

and thus

$$
v^{\prime}(a)+c(\tau) v(a)=\frac{1}{2}\left(\frac{c(\tau)}{\tau}-1\right) e^{-\tau(a-b)} \int_{b-\epsilon}^{b} e^{-\tau(b-y)} f(y) d y .
$$

Next we compute $w(a)$. Since $w \in L^{2}(-\infty, a)$ one can write

$$
w(x, \tau)=\frac{1}{2 \tau} \int_{-\infty}^{a} e^{-\tau|x-y|}\left(f(y)-e^{-\tau T} F(y, \tau)\right) d y+A e^{\tau(x-a)},
$$

where $A$ is an undetermined constant. From this we have

$$
\begin{gathered}
w^{\prime}(x, \tau)=-\frac{1}{2}\left(\int_{-\infty}^{x} e^{-\tau(x-y)}\left(f(y)-e^{-\tau T} F(y, \tau)\right) d y-\int_{x}^{a} e^{-\tau(y-x)}\left(f(y)-e^{-\tau T} F(y, \tau)\right) d y\right) \\
+\tau A e^{\tau(x-a)} .
\end{gathered}
$$

Substituting (4.11) and (4.12) into (4.3), one gets

$$
\begin{gathered}
-\frac{1}{2} \int_{-\infty}^{a} e^{-\tau(a-y)}\left(f(y)-e^{-\tau T} F(y, \tau)\right) d y+\tau A \\
+c(\tau)\left(\frac{1}{2 \tau} \int_{-\infty}^{a} e^{-\tau(a-y)}\left(f(y)-e^{-\tau T} F(y, \tau)\right) d y+A\right)+e^{-\tau T} g(\tau)=0
\end{gathered}
$$

From this we obtain

$$
\begin{gathered}
A=-\frac{1}{2(c(\tau)+\tau)}\left(\frac{c(\tau)}{\tau}-1\right) \int_{-\infty}^{a} e^{-\tau(a-y)} f(y) d y \\
+\frac{e^{-\tau T}}{2(c(\tau)+\tau)}\left(\frac{c(\tau)}{\tau}-1\right) \int_{-\infty}^{a} e^{-\tau(a-y)} F(y, \tau) d y-\frac{e^{-\tau T}}{c(\tau)+\tau} g(\tau)
\end{gathered}
$$

and (4.11) yields

$$
\begin{gathered}
w(a, \tau) \\
=\frac{1}{c(\tau)+\tau} \int_{-\infty}^{a} e^{-\tau(a-y)} f(y) d y-\frac{e^{-\tau T}}{c(\tau)+\tau}\left(\int_{-\infty}^{a} e^{-\tau(a-y)} F(y, \tau) d y+g(\tau)\right) .
\end{gathered}
$$


From (4.4), we see that the integral of the second term of (4.13) has the bound $O\left(\tau^{1 / 2}\right)$. From this together with the trivial equality

$$
\int_{-\infty}^{a} e^{-\tau(a-y)} f(y) d y=e^{-\tau(a-b)} \int_{-\infty}^{a} e^{-\tau(b-y)} f(y) d y
$$

we obtain

$$
w(a, \tau)=\frac{e^{-\tau(a-b)}}{c(\tau)+\tau} \int_{-\infty}^{a} e^{-\tau(b-y)} f(y) d y+O\left(e^{-\tau T} \tau^{-1 / 2}\right) .
$$

Now a combination of (4.5), (4.6), (4.9), (4.10) and (4.14) gives (4.8).

Here we introduce another important assumption on $f$ which means, implicitly, that $x=b$ is not a zero point of $f(x)$ with infinite order:

$$
\exists \mu \in \mathbf{R} \liminf _{\tau \longrightarrow \infty} \tau^{\mu}\left|\int_{b-\epsilon}^{b} e^{-\tau(b-y)} f(y) d y\right|>0 .
$$

Having (4.8), one can easily obtain the following theorem.

Theorem 4.1. Let $f$ satisfy (4.5) and (4.15). Let $T>2 \operatorname{dist}(D, B)-\operatorname{dist}(\Omega, B)$.

(i) Assume that $\gamma \neq 1$. We have:

if $\gamma<1$, then there exists a $\tau_{0}>0$ such that, for all $\tau \geq \tau_{0}$

$$
I(\tau)>0
$$

if $\gamma>1$, then there exists a $\tau_{0}>0$ such that, for all $\tau \geq \tau_{0}$

$$
I(\tau)<0
$$

In both cases we have

$$
\lim _{\tau \longrightarrow \infty} \frac{1}{2 \tau} \log |I(\tau)|=-\operatorname{dist}(D, B) .
$$

(ii) Assume that $\gamma=1$ and $\beta \neq 0$. We have:

if $\beta<0$, then there exists a $\tau_{0}>0$ such that, for all $\tau \geq \tau_{0}$

$$
I(\tau)>0
$$

if $\beta>0$, then there exists a $\tau_{0}>0$ such that, for all $\tau \geq \tau_{0}$

$$
I(\tau)<0
$$

In both cases we have (4.16).

Similarly to (2.51), one has

$$
\int_{B} f(w-v) d y=I(\tau)+O\left(\tau^{-1} e^{-\tau T}\right) .
$$

This connects two types of data asymptotically.

A combination of (4.8) and (4.17) yields the following theorem for back-scattering case. 
Theorem 4.2. Let $f$ satisfy (4.5) and (4.15). Let $T>2 \operatorname{dist}(D, B)$.

(i) Assume that $\gamma \neq 1$. We have:

if $\gamma<1$, then there exists a $\tau_{0}>0$ such that, for all $\tau \geq \tau_{0}$

$$
\int_{B} f(w-v) d y>0
$$

if $\gamma>1$, then there exists a $\tau_{0}>0$ such that, for all $\tau \geq \tau_{0}$

$$
\int_{B} f(w-v) d y<0
$$

In both cases we have

$$
\lim _{\tau \longrightarrow \infty} \frac{1}{2 \tau} \log \left|\int_{B} f(w-v) d y\right|=-\operatorname{dist}(D, B) .
$$

(ii) Assume that $\gamma=1$ and $\beta \neq 0$. We have:

if $\beta<0$, then there exists a $\tau_{0}>0$ such that, for all $\tau \geq \tau_{0}$

$$
\int_{B} f(w-v) d y>0
$$

if $\beta>0$, then there exists a $\tau_{0}>0$ such that, for all $\tau \geq \tau_{0}$

$$
\int_{B} f(w-v) d y<0
$$

In both cases we have (4.18).

Thus it is interesting to consider what happens in the case when $\gamma=1$ and $\beta=0$.

In this case from (4.8) we see that $e^{2 \tau \operatorname{dist}(D, B)} I(\tau)$ is exponentially decaying as $\tau \longrightarrow$ $\infty$ provided $T>2 \operatorname{dist}(D, B)-\operatorname{dist}(\Omega, B)$. Thus it seems that one has to study more about the remainder term hidden in $(4.8)$ by $O\left(e^{-\tau(T+\operatorname{dist}(D, B))}+\tau e^{-\tau(T+\operatorname{dist}(\Omega, B))}\right)$. However, in fact, we see that $u(x, t)$ and thus $I(\tau)$ are independent of $a$ by the following argument.

Given $f \in L^{2}(\mathbf{R})$ with compact support define

$$
u(x, t)=\frac{1}{2} \int_{x-t}^{x+t} f(y) d y .
$$

We see that

$$
\partial_{t} u+\partial_{x} u=f(x+t)
$$

Thus if $\operatorname{supp} f \subset]-\infty, a]$, then $f(a+t)=0$ for any $t>0$ and thus $u$ satisfies $-\partial_{x} u(a, t)-$ $\partial_{t} u(a, t)=0, t>0$. Of course $u$ satisfies the wave equation in the whole space and the initial conditions $u(x, 0)=0, \partial_{t} u(x, 0)=f(x)$. Therefore by the uniqueness of the weak solution of $(4.1)$ we have $u(x, t)=u_{f}(x, t)$ for $\left.(x, t) \in\right]-\infty, a[\times] 0, T[$. However it is easy to see that the right-hand side of (4.19) is independent of $a$ provided supp $f \subset]-\infty, 0[$.

Summing up, we conclude:

- if $\gamma=1$ and $\beta=0$, then $D$ is invisible by the data $u_{f}(0, t)$ for $0<t<T$ or $u_{f}(x, t)$ for $(x, t) \in B \times] 0, T[$ for any $T$ and $f$ with $\sup f \subset]-\infty, 0[$.

Finally we note that one can also determine $\gamma$ and $\beta$ after having known dist $(D, B)$. More precisely we have the following formula. 
Theorem 4.3. Let $f$ satisfy (4.5) and (4.15).

(i) If $T>2 \operatorname{dist}(D, B)-\operatorname{dist}(\Omega, B)$, then as $\tau \longrightarrow \infty$ we have the following complete asymptotic expansion:

$$
\frac{e^{2 \tau \operatorname{dist}(D, B)} I(\tau)}{\left|\int_{b-\epsilon}^{b} e^{-\tau(b-y)} f(y) d y\right|^{2}} \sim-\frac{\gamma-1}{2(\gamma+1)} \frac{1}{\tau}-\frac{\beta}{(\gamma+1)^{2}} \sum_{n=0}^{\infty}\left(-\frac{\beta}{\gamma+1}\right)^{n} \frac{1}{\tau^{n+2}}
$$

(ii) If $T>2 \operatorname{dist}(D, B)$, then as $\tau \longrightarrow \infty$ the function

$$
\tau \longmapsto \frac{e^{2 \tau \operatorname{dist}(D, B)} \int_{B} f(w-v) d y}{\left|\int_{b-\epsilon}^{b} e^{-\tau(b-y)} f(y) d y\right|^{2}}
$$

has the same asymptotic expansion as (4.20).

Proof. (4.15) means that there exist a positive constant $C$ and $\tau_{0}>0$ such that for all $\tau \geq \tau_{0}$

$$
\left|\int_{b-\epsilon}^{b} e^{-\tau(b-y)} f(y) d y\right| \geq C \tau^{-\mu} .
$$

This together with (4.8) gives

$$
\begin{gathered}
\frac{e^{2 \tau \operatorname{dist}(D, B)} I(\tau)}{\left|\int_{b-\epsilon}^{b} e^{-\tau(b-y)} f(y) d y\right|^{2}}=-\frac{1}{2 \tau} \frac{(\gamma-1) \tau+\beta}{(\gamma+1) \tau+\beta} \\
+O\left(\tau^{2 \mu} e^{-\tau(T-\operatorname{dist}(D, B))}+\tau^{2 \mu+1} e^{-\tau(T-2 \operatorname{dist}(D, B)+\operatorname{dist}(\Omega, B))}\right) .
\end{gathered}
$$

Thus expanding the first term of this right-hand side as $\tau \longrightarrow \infty$, we obtain $(4.20)$.

(ii) is a direct consequence of (4.17), (4.21) and (i).

Note that from the coefficients of the first and second terms of the right-hand side in (4.20) one gets $\gamma$ and successively $\beta$,

\subsection{Some open problems}

- To study the asymptotic behaviour of (1.21) as $\tau \longrightarrow \infty$ as done in Theorem 4.3 in one-space dimensional case. We think that in three dimensions some restriction of the geometry about $D$ should be imposed and have to use (2.14) or (2.52) instead of (2.15). For the purpose the asymptotic profile of $w$ on $\partial D$ or $B$ has to be clarified as done in one space dimensional case, see (4.14)

- The case when $\gamma=1$ and $\beta=0$ seems pathological, however, from a mathematical point of view it would be interesting to study the asymptotic behaviour of $I(\tau)$ as $\tau \longrightarrow \infty$ in three-dimensions since in one-space dimensional case one can not extract the distance of $B$ to $D$. 
When the data is given by the back-scattering kernel in the Lax-Phillips scattering theory, a corresponding positive result in three dimensions has been announced in Georgiev-Arnaoudov [2].

Note also that Hansen [5] considered a corresponding problem in the Lax-Phillips scattering theory for a transparent obstacle with a smooth $\alpha$ on $\bar{D}$ whose trace onto $\partial D$ is 1 , however, its normal derivative on $\partial D$ is nonzero everywhere. This is the case when both of (B1) and (B2) are not satisfied. He showed that the back-scattering kernel still catches the values of the support function of $D$ as the leading singularity. What can one say in our problem setting?

- Can one say something when the data are measured on another fixed ball $B^{\prime}$ with $\bar{B}^{\prime} \cap \bar{D}=\emptyset$ and $\bar{B}^{\prime} \cap \bar{B}=\emptyset$ ? For example, what happens on the asymptotic behaviour of the following integral involving $f$ and another function $f^{\prime}$ with supp $f^{\prime}=\bar{B}^{\prime}$ as $\tau \longrightarrow \infty$ :

$$
\int_{B^{\prime}} f^{\prime}\left(w_{f}-v_{f}\right) d x .
$$

We think that this is a model of the case when the emitter and receivers of the signal are placed on different positions and what we can use is just a single pair of the emitter and receiver.

- It would be interesting to test the performance of formulae (1.7), (1.12), (1.17) an (1.20) by using numerically simulated data.

- Expand the range of applications of the method presented in [9] and here to other prototype inverse obstacle scattering problems for acoustic/electromagentic/elastic waves or their couplings, etc. In the framework of the Lax-Phillips scattring theory there is a result by Georgiev [3] for a moving obstacle. How can one treate the case in our framework?

\section{Acknowledgment}

The author was partially supported by Grant-in-Aid for Scientific Research (C)(No. 21540162) of Japan Society for the Promotion of Science.

\section{References}

[1] Dautray, R. and Lions, J-L., Mathematical analysis and numerical methods for sciences and technology, Evolution problems I, Vol. 5, Springer-Verlag, Berlin, 1992.

[2] Georgiev, V. and Arnaoudov, Ya., Inverse scattering problem for dissipative wave equation, Integral Equations and Inverse Problems, Pitman Research Notes in Mathematics Series, 235, 86-89, Longman, 1991.

[3] Georgiev, V., Inverse scattering problem for the Maxwell equation outside moving body, Ann. Inst. Henri Poincaré, 50(1989), no. 1, 37-70.

[4] Grisvard, P., Elliptic problems in nonsmooth domains, Pitman, Boston, 1985. 
[5] Hansen, S., The leading singularity of the scattering kernel for a transparent obstacle, Math. Ann., 279(1987), 125-139.

[6] Ikehata, M., Enclosing a polygonal cavity in a two-dimensional bounded domain from Cauchy data, Inverse Problems, 15(1999), 1231-1241.

[7] Ikehata, M., Reconstruction of the support function for inclusion from boundary measurements, J. Inv. Ill-Posed Problems, 8(2000), 367-378.

[8] Ikehata, M., Extracting discontinuity in a heat conductive body. One-space dimensional case, Applicable Analysis, 86(2007), no. 8, 963-1005.

[9] Ikehata, M., The enclosure method for inverse obstacle scattering problems with dynamical data over a finite time interval, Inverse Problems, 26(2010) 055010(20pp).

[10] Ikehata, M., The framework of the enclosure method with dynamical data and its applications, Inverse Problems, 27(2011) 065005(16pp).

[11] Ikehata, M. and Kawashita, M., The enclosure method for the heat equation, Inverse Problems, 25(2009) 075005(10pp).

[12] Ikehata, M. and Kawashita, M., On the reconstruction of inclusions in a heat conductive body from dynamical boundary data over a finite time interval, Inverse Problems, 26(2010) 095004(15pp).

[13] Lax, P. D. and Phillips, R. S., Scattering theory for dissipative hyperbolic systems, J. Func. Analysis, 14(1973), 172-235.

[14] Majda, A., High frequency asymptotics for the scattering matrix and the inverse problem of acoustic scattering, Comm. Pure and Appl. Math., 29(1976), 261-291.

[15] Majda, A. and Taylor, M., Inverse scattering problems for transparent obstacles, Comm. in partial differential equations, 2(4)(1977), 395-438.

[16] Petkov, V., Propagation of sigularities and inverse scattering problem for transparent obstacles, J. Math. Pures Appl., 60(1982), 65-90.

[17] Petkov, V., Inverse scattering problem for transparent obstacles, Math. Proc. Camb. Phil. Soc., 92(1982), 361-367.

e-mail address

ikehata@math.sci.gunma-u.ac.jp 